Groups Geom. Dyn. 5 (2011), 691-728

DOI $10.4171 / \mathrm{GGD} / 145$
Groups, Geometry, and Dynamics

(C) European Mathematical Society

\title{
Characterizing the Cantor bi-cube in asymptotic categories
}

\author{
Taras Banakh and Ihor Zarichnyi
}

\begin{abstract}
We present characterizations of metric spaces that are micro-, macro- or bi-uniformly equivalent to the extended Cantor set $\mathrm{EC}=\left\{\sum_{i=-n}^{\infty} \frac{2 x_{i}}{3^{i}} \mid n \in \mathbb{N},\left(x_{i}\right)_{i \in \mathbb{Z}} \in\{0,1\}^{\mathbb{Z}}\right\} \subset$ $\mathbb{R}$, which is bi-uniformly equivalent to the Cantor bi-cube $2^{<\mathbb{Z}}=\left\{\left(x_{i}\right)_{i \in \mathbb{Z}} \in\{0,1\}^{\mathbb{Z}} \mid\right.$ there exists $n$ such that $x_{i}=0$ for all $i \geq n$ \} endowed with the metric $d\left(\left(x_{i}\right),\left(y_{i}\right)\right)=$ $\max _{i \in \mathbb{Z}} 2^{i}\left|x_{i}-y_{i}\right|$. The characterizations imply that any two (uncountable) proper isometrically homogeneous ultrametric spaces are coarsely (and bi-uniformly) equivalent. This implies that any two countable locally finite groups endowed with proper left-invariant metrics are coarsely equivalent. For the proof of these results we develop a technique of towers which may be of independent interest.
\end{abstract}

Mathematics Subject Classification (2010). 54E35, 54E40.

Keywords. Locally finite groups, coarse equivalence, ultrametric spaces, extended Cantor set.

\section{Introduction}

This paper is motivated by the problem of coarse classification of countable locally finite groups posed in [BDHM], repeated in [Sj], Problem 1606, and communicated to the authors by I. V. Protasov. As we will see later, a crucial role in this classification is played by the extended Cantor set

$$
\mathrm{EC}=\left\{\sum_{i=-n}^{\infty} \frac{2 x_{i}}{3^{i}} \mid n \in \mathbb{N},\left(x_{i}\right)_{i \in \mathbb{Z}} \in\{0,1\}^{\mathbb{Z}}\right\} \subset \mathbb{R} .
$$

Firstly we present four characterizations of the extended Cantor set EC in various categories of metric spaces and then we apply these characterizations to the problem of coarse and bi-uniform classifications of locally finite groups (more generally of isometrically homogeneous metric spaces).

We will mainly work in the categories of proper metric spaces and their (macro-, micro-, or bi-) uniform maps. It will be convenient to introduce such maps using the notion of the oscillation $\omega_{f}$ of a function $f: X \rightarrow Y$ between metric spaces $X$ and $Y$. By definition, the oscillation of $f$ is the function $\omega_{f}:[0, \infty) \rightarrow[0, \infty]$ assigning to each $\delta \geq 0$ the (finite or infinite) number

$$
\omega_{f}(\delta)=\sup \left\{\operatorname{dist}\left(f(x), f\left(x^{\prime}\right)\right) \mid x, x^{\prime} \in X, \operatorname{dist}\left(x, x^{\prime}\right) \leq \delta\right\} .
$$


Here $\operatorname{dist}\left(x, x^{\prime}\right)$ denotes the distance between points $x, x^{\prime}$ in a metric space.

A map $f: X \rightarrow Y$ is called

- uniformly continuous (or else micro-uniform) if for any $\varepsilon>0$ there exists $\delta>0$ with $\omega_{f}(\delta) \leq \varepsilon$

- macro-uniform if for any $\delta<\infty$ there exists $\varepsilon<\infty$ with $\omega_{f}(\delta) \leq \varepsilon$;

- bi-uniform if $f$ is macro- and micro-uniform.

These notions induce the corresponding equivalences of metric spaces. Namely, a map $f: X \rightarrow Y$ between two metric spaces is called

- a uniform homeomorphism if $f$ is bijective and both $f$ and $f^{-1}$ are uniformly continuous;

- a bi-uniform equivalence if $f$ is bijective and both $f$ and $f^{-1}$ are bi-uniform maps;

- a coarse equivalence if $f$ is macro-uniform and there exists a macro-uniform map $g: Y \rightarrow X$ such that $\operatorname{dist}\left(f \circ g, \operatorname{id}_{Y}\right)<\infty$ and $\operatorname{dist}\left(g \circ f, \operatorname{id}_{X}\right)<\infty$.

Observe that a map $f: X \rightarrow Y$ is a bi-uniform equivalence if and only if $f$ is both a uniform homeomorphism and a coarse equivalence.

We have defined morphisms and isomorphisms in our categories and now switch to the objects.

We say that a metric space $X$

- is isometrically homogeneous if for any two points $x, y \in X$ there is a bijective isometry $f: X \rightarrow X$ such that $f(x)=y$;

- is proper if $X$ is unbounded, but for every $x_{0} \in X$ and $r \in[0,+\infty)$ the closed $r$-ball $B_{r}\left(x_{0}\right)=\left\{x \in X \mid \operatorname{dist}\left(x, x_{0}\right) \leq r\right\}$ centered at $x_{0}$ is compact;

- has bounded geometry if there is $\delta<\infty$ such that for every $\varepsilon<\infty$ there exists $n \in \mathbb{N}$ such that each $\varepsilon$-ball in $X$ can be covered by $\leq n$ balls of radius $\delta$;

- is ultrametric if $d(x, y) \leq \max \{d(x, z), d(z, y)\}$ for any points $x, y, z \in X$.

Ultrametric spaces often appear as natural examples of zero-dimensional spaces (in various senses), see [BDHM]. We are interested in four notions of zero-dimensionality: topological, micro-uniform, macro-uniform (= asymptotic), and biuniform.

First, given a positive real number $s$ define the $s$-connected component of a point $x$ of a metric space $X$ as the set $C_{s}(x)$ of all points $y \in X$ that can be linked with $x$ by a chain of points $y=z_{0}, z_{1}, \ldots, z_{n}=x \operatorname{such}$ that $\operatorname{dist}\left(z_{i-1}, z_{i}\right) \leq s$ for all $i \leq n$. By $\ell_{s}(X)=\left\{C_{S}(x) \mid x \in X\right\}$ we denote the family of the (pairwise disjoint) $s$-connected components of $X$. Given a family $\ell$ of subsets of a metric space $X$ let

$$
\operatorname{mesh} \mathcal{C}=\sup _{C \in \mathcal{C}} \operatorname{diam}(C)
$$

For a metric space $X$ and positive real numbers $\delta \leq \varepsilon$ consider the cardinal 
characteristics

$$
\theta_{\delta}^{\varepsilon}(X)=\min _{x \in X}\left|C_{\varepsilon}(x) / \mathscr{C}_{\delta}(X)\right| \quad \text { and } \quad \Theta_{\delta}^{\varepsilon}(X)=\sup _{x \in X}\left|C_{\varepsilon}(x) / \mathscr{C}_{\delta}(X)\right|,
$$

where $\left|C_{\varepsilon}(x) / \mathscr{C}_{\delta}(X)\right|=\left|\left\{C_{\delta}(y) \mid y \in C_{\varepsilon}(x)\right\}\right|$ is the number of $\delta$-connected components composing the $\varepsilon$-connected component $C_{\varepsilon}(x)$ of $x$.

If the metric space $X$ is isometrically homogeneous, then $\theta_{\delta}^{\varepsilon}(X)=\Theta_{\delta}^{\varepsilon}(X)=$ $\left|C_{\varepsilon}(x) / \mathscr{C}_{\delta}(X)\right|$ for every $x \in X$. If $X$ is an ultrametric space, then the $\varepsilon$-connected component $C_{\varepsilon}(x)$ of a point $x$ coincides with the closed $\varepsilon$-ball $B_{\varepsilon}(x)$ and thus $\left|C_{\varepsilon}(x) / \mathscr{C}_{\delta}(X)\right|$ is just the number of $\delta$-balls composing the $\varepsilon$-ball $B_{\varepsilon}(x)$. Observe that an ultrametric space $X$ has bounded geometry if and only if there is $\delta<\infty$ such that $\Theta_{\delta}^{\varepsilon}(X)$ if finite for every finite $\varepsilon \geq \delta$.

We say that a metric space $X$ has

- topological dimension zero if the family of closed-and-open subsets forms a base of the topology of $X$;

- micro-uniform dimension zero if for all $\varepsilon>0$ there exists $\delta>0$ with $\operatorname{mesh} \boldsymbol{C}_{\delta}(X) \leq \varepsilon$

- macro-uniform (or else asymptotic) dimension zero if for all $\delta<\infty$ there exists $\varepsilon<\infty$ with mesh $\bigodot_{\delta}(X) \leq \varepsilon$;

- bi-uniform dimension zero if $X$ has both micro-uniform and macro-uniform dimensions zero.

It follows that a metric space $X$ of bi-uniform dimension zero has topological, microuniform, and macro-uniform dimensions zero.

If $X$ is an ultrametric space, then for every $s>0$ the $s$-connected component $C_{s}(x)$ of a point $x \in X$ coincides with the closed $s$-ball $B_{s}(x)$. So $X$ has bi-uniform dimension zero (because mesh $\ell_{s}(X)=s$ for all $s>0$ ). On the other hand, each metric space of asymptotic (bi-uniform) dimension zero is coarsely (bi-uniformly) equivalent to an ultrametric space; see Theorem 4.3 of [BDHM].

The class of proper metric spaces of bi-uniform dimension zero contains an interesting object

$$
\mathrm{EC}=\left\{\sum_{i=-n}^{\infty} \frac{2 x_{i}}{3^{i}} \mid\left(x_{i}\right)_{i \in \mathbb{Z}} \in\{0,1\}^{\mathbb{Z}}, n \in \mathbb{N}\right\} \subset \mathbb{R}
$$

called the extended Cantor set. The extended Cantor set EC coincides with the image of the Cantor bi-cube

$$
2^{<\mathbb{Z}}=\left\{\left(x_{i}\right)_{i \in \mathbb{Z}} \in\{0,1\}^{\mathbb{Z}} \mid \text { there exists } n \in \mathbb{N} \text { such that } x_{i}=0 \text { for all } i>n\right\}
$$

under the map

$$
f: 2^{<\mathbb{Z}} \rightarrow \text { EC, } \quad f:\left(x_{i}\right)_{i \in \mathbb{Z}} \mapsto \sum_{i=-\infty}^{\infty} 2 \cdot 3^{i} \cdot x_{i}
$$


This map determines a bi-uniform equivalence between the extended Cantor set EC and the Cantor bi-cube $2^{<\mathbb{Z}}$ endowed with the ultrametric

$$
d\left(\left(x_{i}\right),\left(y_{i}\right)\right)=\max _{i \in \mathbb{Z}} 2^{i}\left|x_{i}-y_{i}\right| .
$$

The Cantor bi-cube can be written as the product $2^{<\mathbb{Z}}=2^{\omega} \times 2^{<\mathbb{N}}$ of the Cantor micro-cube

$$
2^{\omega}=\left\{\left(x_{i}\right)_{i \in \mathbb{Z}} \in 2^{<\mathbb{Z}} \mid x_{i}=0 \text { for all } i>0\right\}
$$

and the Cantor macro-cube

$$
2^{<\mathbb{N}}=\left\{\left(x_{i}\right)_{i \in \mathbb{Z}} \in 2^{<\mathbb{Z}} \mid x_{i}=0 \text { for all } i \leq 0\right\} .
$$

The Cantor micro-cube can be identified with the standard Cantor cube $\{0,1\}^{\omega}$. It is well known that the Cantor micro-cube $2^{\omega}$ contains a micro-uniform copy of each zero-dimensional compact metric space [Ke], Theorem 7.8. The Cantor macro-cube $2^{<\mathbb{N}}$ has a similar property: it contains a macro-uniform copy of each asymptotically zero-dimensional metric space of bounded geometry; see Theorem 3.11 of [DZ]. This picture is completed by the following result.

Theorem 1 (Universality of the Cantor bi-cube). A metric space $X$ is bi-uniformly equivalent to a subspace of the Cantor bi-cube $2^{<\mathbb{Z}}$ if and only if $X$ is a metric space of bi-uniform dimension zero such that $\Theta_{\delta}^{\varepsilon}(X)<\infty$ for all $0<\delta \leq \varepsilon<\infty$.

Now we turn to the problem of characterization of the spaces $2^{\omega}, 2^{<\mathbb{N}}$, and $2^{<\mathbb{Z}}$ in various categories. The characterization of the Cantor micro-cube is well known and is due to Brouwer (see [Ke], Theorem 7.4):

Theorem 2 (Topological characterization of the Cantor cube). For a metric space $X$ the following conditions are equivalent:

(1) $X$ is topologically equivalent to the Cantor micro-cube $2^{\omega}$;

(2) $X$ is micro-uniformly equivalent to $2^{\omega}$;

(3) $X$ is bi-uniformly equivalent to $2^{\omega}$;

(4) $X$ is a zero-dimensional metric compact space without isolated points.

Since the Cantor bi-cube $2^{<\mathbb{Z}}=2^{\omega} \times 2^{<\mathbb{N}}$, and the Cantor macro-cube $2^{<\mathbb{N}}$ is discrete, the preceding theorem implies the following (well-known) topological characterization of the Cantor bi-cube $2^{<\mathbb{Z}}$ :

Theorem 3 (Topological characterization of the Cantor bi-cube). A metric space $X$ is topologically equivalent to the Cantor bi-cube $2^{<\mathbb{Z}}$ if and only if

(1) $X$ has topological dimension zero;

(2) $X$ is separable, locally compact and non-compact; 
(3) X has no isolated points.

In the next three theorems we present characterizations of the Cantor bi-cube in the micro-, macro-, and bi-uniform categories.

Theorem 4 (Micro-uniform characterization of the Cantor bi-cube). A metric space $X$ is micro-uniformly equivalent to the Cantor bi-cube $2^{<\mathbb{Z}}$ if and only if

(1) $X$ is a non-compact complete metric space of micro-uniform dimension zero;

(2) there exists an $\varepsilon>0$ such that $\Theta_{\delta}^{\varepsilon}(X)$ is finite for all positive $\delta \leq \varepsilon$ and $\lim _{\delta \rightarrow+0} \theta_{\delta}^{\varepsilon}(X)=\infty$.

Theorem 5 (Macro-uniform characterization of the Cantor bi-cube). A metric space $X$ is macro-uniformly equivalent to the Cantor bi-cube $2^{<\mathbb{Z}}$ if and only if

(1) $X$ has macro-uniform dimension zero;

(2) there exists an $\delta>0$ such that $\Theta_{\delta}^{\varepsilon}(X)$ is finite for all positive $\varepsilon \geq \delta$ and $\lim _{\varepsilon \rightarrow \infty} \theta_{\delta}^{\varepsilon}(X)=\infty$.

Theorem 6 (Bi-uniform characterization of the Cantor bi-cube). A metric space $X$ is bi-uniformly equivalent to the Cantor bi-cube $2^{<\mathbb{Z}}$ if and only if

(1) $X$ is a complete metric space of bi-uniform dimension zero;

(2) $\Theta_{\delta}^{\varepsilon}(X)$ is finite for all $0<\delta \leq \varepsilon<\infty$;

(3) $\lim _{\varepsilon \rightarrow \infty} \theta_{\delta}^{\varepsilon}(X)=\infty$ for all $\delta<\infty$;

(4) $\lim _{\delta \rightarrow+0} \theta_{\delta}^{\varepsilon}(X)=\infty$ for all $\varepsilon>0$.

It is clear that any metric space $X$ that is bi-uniformly equivalent to the Cantor bicube $2^{<\mathbb{Z}}$ is micro-uniformly and macro-uniformly equivalent to $2^{<\mathbb{Z}}$. The converse is not true.

Example 1. Let $\omega$ be the space of finite ordinals, endowed with the discrete 2-valued metric. The metric space $2^{\omega} \times \omega \times 2^{<\mathbb{N}}$ is micro-uniformly and macro-uniformly equivalent to $2^{<\mathbb{Z}}$ but fails to be bi-uniformly equivalent to $2^{<\mathbb{Z}}$.

Characterization Theorems 3-6 of the Cantor bi-cube allows us to detect copies of $2^{<\mathbb{Z}}$ among isometrically homogeneous metric spaces:

Corollary 7. An isometrically homogeneous metric space $X$ is

(1) micro-uniformly equivalent to $2^{<\mathbb{Z}}$ if and only if $X$ is homeomorphic to $2^{<\mathbb{Z}}$ if and only if $X$ is uncountable, separable, locally compact, non-compact, and has topological dimension zero;

(2) macro-uniformly equivalent to $2^{<\mathbb{Z}}$ if and only if $X$ is unbounded, has bounded geometry and has asymptotic dimension zero; 
(3) bi-uniformly equivalent to $2^{<\mathbb{Z}}$ if and only if $X$ is proper, uncountable, and has bi-uniform dimension zero.

Now we apply this classification result to the macro- and bi-uniform classification of countable groups, viewed as metric spaces endowed with perfect left-invariant metrics. J. Smith [Sm] observed that each countable group carries a perfect left-invariant metric and such a metric is unique up to the bi-uniform equivalence. A. Dranishnikov and J. Smith [DS] proved that a countable group $G$ endowed with a proper left-invariant metric has asymptotic dimension zero if and only if $G$ is locally finite in the sense that each finitely-generated subgroup of $G$ is finite. The authors of [BDHM] classified countable locally finite groups up to the bi-uniform equivalence and posed the problem of classification of countable locally finite groups up to the coarse equivalence. The same problem was repeated by J. Sanjurjo in [Sj], Problem 1606. The following corollary of Corollary 7 (2) answers this problem.

Corollary 8. Any two countable locally finite groups endowed with proper leftinvariant metrics are coarsely equivalent.

This corollary is a principal ingredient in the coarse classification of countable abelian groups given in [BHZ].

Corollary 7 shows that the coarse classification of proper isometrically homogeneous metric spaces of asymptotic dimension zero is trivial: all such spaces are coarsely equivalent. The same concerns the bi-uniform classification of uncountable proper isometrically homogeneous metric spaces of bi-uniform dimension zero: all such spaces are bi-uniformly equivalent. Also the micro-uniform classification of countable proper isometrically homogeneous metric spaces is trivial: all such spaces are micro-uniformly equivalent to $\mathbb{Z}$. In contrast, the bi-uniform classification of countable proper isometrically homogeneous metric spaces of uniform dimension zero is non-trivial and yields continuum many non-equivalent spaces.

First observe that Baire's theorem guarantees that each countable proper isometrically homogeneous metric space $X$ is boundedly-finite in the sense that all bounded subsets of $X$ are finite.

For each boundedly-finite metric space $X$ of asymptotic dimension zero we can consider the function $f_{X}: \Pi \rightarrow \omega \cup\{\infty\}$ defined on the set $\Pi$ of prime numbers and assigning to each $p \in \Pi$ the number

$$
f_{X}(p)=\sup \left\{n \in \omega \mid p^{n} \text { divides }\left|C_{s}(x)\right| \text { for some } x \in X \text { and } s>0\right\},
$$

where $C_{s}(x)$ stands for the $s$-connected component of $x$. It turns out that the function $f_{X}$ completely determines the bi-uniform type of a countable proper isometrically homogeneous metric space $X$ of asymptotic dimension zero.

Theorem 9. Two countable proper isometrically homogeneous metric spaces $X, Y$ of asymptotic dimension zero are bi-uniformly equivalent if and only if $f_{X}=f_{Y}$. 
For countable groups (endowed with proper left-invariant metrics) Theorem 9 has been proved in [BDHM].

Observe that for any function $f: \Pi \rightarrow \omega \cup\{\infty\}$ there is a countable proper isometrically homogeneous ultrametric space $X$ with $f=f_{X}$. Indeed, consider the abelian group

$$
\mathbb{Z}_{f}=\bigoplus_{p \in \Pi} \mathbb{Z}_{p}^{f(p)}
$$

If $f(p)=\infty$ then $\mathbb{Z}_{p}^{f(p)}=\mathbb{Z}_{p}^{\infty}$ is the direct sum of countably many copies of the cyclic group $\mathbb{Z}_{p}=\mathbb{Z} / p \mathbb{Z}$. Endowing the group $\mathbb{Z}_{f}$ with a suitable proper left-invariant metric $d$, we can see that the metric space $X=\left(\mathbb{Z}_{f}, d\right)$ has $f_{X}=f$. Combining this observation with Corollary 7 (3) and Theorem 9, we get the following bi-uniform classification of proper isometrically homogeneous metric spaces of biuniform dimension zero.

Corollary 10. A proper isometrically homogeneous metric space $X$ of bi-uniform dimension zero is bi-uniformly equivalent to

- the Cantor bi-cube $2^{<\mathbb{Z}}$ if $X$ is uncountable;

- the group $\mathbb{Z}_{f_{X}}$ if $X$ is countable.

\section{Characterizing the coarse equivalence}

In this section we show that various natural ways of defining morphisms in asymptology ${ }^{1}$ lead to the same notion of coarse equivalence. Besides the original approach of J. Roe [Roe] based on the notion of a coarse map, we discuss an alternative approach based on the notion of a multi-map.

By a multi-map $\Phi: X \Rightarrow Y$ between two sets $X, Y$ we understand any subset $\Phi \subset X \times Y$.

For a subset $A \subset X$ by $\Phi(A)=\{y \in Y \mid$ there exists $a \in A$ with $(a, y) \in \Phi\}$ we denote the image of $A$ under the multi-map $\Phi$. Given a point $x \in X$ we write $\Phi(x)$ instead of $\Phi(\{x\})$.

The inverse $\Phi^{-1}: Y \Rightarrow X$ to the multi-map $\Phi$ is the subset $\Phi^{-1}=\{(y, x) \in$ $Y \times X \mid(x, y) \in \Phi\} \subset Y \times X$. For two multi-maps $\Phi: X \Rightarrow Y$ and $\Psi: Y \Rightarrow Z$ we define their composition $\Psi \circ \Phi: X \Rightarrow Z$ as usual:

$\Psi \circ \Phi=\{(x, z) \in X \times Z \mid$ there exists $y \in Y$ such that $(x, y) \in \Phi$ and $(y, z) \in \Psi\}$.

A multi-map $\Phi$ is called surjective if $\Phi(X)=Y$ and bijective if $\Phi \subset X \times Y$ coincides with the graph of a bijective (single-valued) function.

\footnotetext{
${ }^{1}$ The term "asymptology" was coined by I. Protasov in [PZ] for naming the theory studying large scale properties of metric spaces (or more general objects like balleans of I. Protasov [PZ], [PB] or coarse structures of J. Roe [Roe]).
} 
The oscillation of a multi-map $\Phi: X \Rightarrow Y$ between metric spaces is the function $\omega_{\Phi}:[0, \infty) \rightarrow[0, \infty]$ assigning to each $\delta \geq 0$ the (finite or infinite) number

$$
\omega_{\Phi}(\delta)=\sup \{\operatorname{diam}(\Phi(A)) \mid A \subset X, \operatorname{diam}(A) \leq \delta\} .
$$

Observe that $\omega_{\Phi}(\Phi)=0$ if and only if $\Phi$ is at most single-valued in the sense that $|\Phi(x)| \leq 1$ for any $x \in X$.

A multi-map $\Phi: X \Rightarrow Y$ between metric spaces $X$ and $Y$ is called

- micro-uniform if for all $\varepsilon>0$ there exists $\delta>0$ with $\omega_{\Phi}(\delta) \leq \varepsilon$;

- macro-uniform if for all $\delta<\infty$ there exists $\varepsilon<\infty$ with $\omega_{\Phi}(\delta) \leq \varepsilon$;

- bi-uniform if $\Phi$ is both micro-uniform and macro-uniform.

A multi-map $\Phi: X \Rightarrow Y$ is called a bi-uniform (resp. micro-uniform, macrouniform) embedding if $\Phi^{-1}(Y)=X$ and both multi-maps $\Phi$ and $\Phi^{-1}$ are bi-uniform (resp. micro-uniform, macro-uniform). If, in addition, $\Phi(X)=Y$, then $\Phi$ is called a bi-uniform (resp. micro-uniform, macro-uniform) equivalence.

Two metric spaces $X, Y$ are called bi-uniformly (resp. micro-uniformly, macrouniformly) equivalent if there is a bi-uniform (resp. micro-uniform, macro-uniform) equivalence $\Phi: X \Rightarrow Y$.

It follows that each micro-uniform multi-map is at most single-valued and thus is uniformly continuous in the usual sense. So, two metric spaces $X, Y$ are microuniformly equivalent if and only if they are uniformly homeomorphic. On the other hand, the notion of bi-uniform equivalence agrees with that given in the introduction. In Proposition 2.1 below we will prove that metric spaces are macro-uniformly equivalent if and only if they are coarsely equivalent.

A subset $L$ of a metric space $X$ is called large if $B_{r}(L)=X$ for some $r \in \mathbb{R}$, where $B_{r}(L)=\{x \in X \mid \operatorname{dist}(x, L) \leq r\}$ stands for the closed $r$-neighborhood of the set $L$ in $X$.

For two multi-maps $\Phi: \Psi: X \Rightarrow Y$ between metric spaces let

$$
\begin{array}{r}
\operatorname{dist}(\Psi, \Phi)=\inf \left\{r \in[0, \infty] \mid \Phi(x) \subset B_{r}(\Psi(x)) \text { and } \Psi(x) \subset B_{r}(\Phi(x))\right. \\
\\
\text { for all } x \in X\} .
\end{array}
$$

The following characterization is the main (and unique) result of this section.

Proposition 2.1. For metric spaces $X, Y$ the following assertions are equivalent:

(1) $X$ and $Y$ are macro-uniformly equivalent;

(2) $X$ and $Y$ are coarsely equivalent;

(3) the spaces $X, Y$ contain bi-uniformly equivalent large subspaces $X^{\prime} \subset X$ and $Y^{\prime} \subset Y$;

(4) there are two macro-uniform maps $f: X \rightarrow Y$ and $g: Y \rightarrow X$, the inverses $f^{-1}: Y \Rightarrow X$ and $g^{-1}: X \Rightarrow Y$ of which are macro-uniform and $\max \left\{\operatorname{dist}\left(g \circ f, \operatorname{id}_{X}\right), \operatorname{dist}\left(f \circ g, \operatorname{id}_{Y}\right)\right\}<\infty$. 
Proof. To prove the equivalence of the items (1)-(4), it suffices to establish the implications (1) $\Rightarrow(4) \Rightarrow(2) \Rightarrow(3) \Rightarrow(1)$.

$(1) \Rightarrow(4)$ : Assuming that $X$ and $Y$ are macro-uniformly equivalent, fix a surjective macro-uniform multi-map $\Phi: X \Rightarrow Y$ with surjective macro-uniform inverse $\Phi^{-1}: Y \Rightarrow X$. Since the multi-map $\Phi^{-1}$ is surjective, for every $x \in X$ the subset $\Phi(x) \subset Y$ is not empty and thus contains some point $f(x) \in \Phi(x)$. It follows from the macro-uniformity of $\Phi$ that the map $f: X \rightarrow Y$ is macro-uniform. Since $f^{-1}(y) \subset \Phi^{-1}(y)$ for all $y \in Y$, the macro-uniformity $\Phi^{-1}$ implies the macrouniformity of the multi-map $f^{-1}: Y \Rightarrow X$.

By the same reason, the surjectivity of the multi-map $\Phi$ implies the existence of a map $g: Y \rightarrow X$ such that $g(y) \in \Phi^{-1}(y)$ for all $y \in Y$. The macro-uniformity $\Phi$ and $\Phi^{-1}$ implies that $g: Y \rightarrow X$ and $g^{-1}: X \Rightarrow Y$ are macro-uniform.

As the composition $\Phi^{-1} \circ \Phi: X \Rightarrow X$ is macro-uniform, there is a constant $C<$ $\infty$ such that diam $\Phi^{-1} \circ \Phi(x) \leq C$ for all $x \in X$. From $\{x, g \circ f(x)\} \subset \Phi^{-1} \circ \Phi(x)$ we see that $\operatorname{dist}\left(g \circ f, \operatorname{id}_{X}\right) \leq C<\infty$. By the same reason, $\operatorname{dist}\left(f \circ g, \operatorname{id}_{Y}\right)<\infty$.

The implication (4) $\Rightarrow$ (2) trivially follows from the definition of the coarse equivalence given in the Introduction.

$(2) \Rightarrow(3)$ : Assume that there are two macro-uniform maps $f: X \rightarrow Y, g: Y \rightarrow$ $X$ with $\operatorname{dist}\left(g \circ f, \mathrm{id}_{X}\right)<R$ and $\operatorname{dist}\left(f \circ g, \mathrm{id}_{Y}\right)<R$ for some real number $R$. It follows that $B_{R}(f(X))=Y$ and hence the set $f(X)$ is large in $Y$. Since $f$ is macro-uniform, the number $S=1+\omega_{f}(1)$ is finite. Let $Y^{\prime} \subset f(X)$ be a maximal $S$ separated subset of $f(X)$. The $S$-separated property of $Y^{\prime}$ means that $\operatorname{dist}\left(y, y^{\prime}\right) \geq S$ for any distinct points $y, y^{\prime} \in Y^{\prime}$. The maximality of $Y^{\prime}$ guarantees that $Y^{\prime}$ is large in $f(X)$ and consequently, in $Y$.

Choose any subset $X^{\prime} \subset X$ making the restriction $h=f \mid X^{\prime}: X^{\prime} \rightarrow Y^{\prime}$ bijective. The map $h$ is macro-uniform, since it is a restriction of a macro-uniform map. The choice of the number $S$ guarantees that the set $X^{\prime}$ is 1-separated and consequently, the map $h$ is micro-uniform. Since $Y^{\prime}$ is $S$-separated the inverse map $h^{-1}: Y^{\prime} \rightarrow X^{\prime}$ is micro-uniform.

It remains to check that $h^{-1}$ is macro-uniform. Given arbitrary $\varepsilon<\infty$, use the macro-uniformity of the map $g: Y \rightarrow X$ to conclude that the number $\delta=\omega_{g}(\varepsilon)$ is finite. Now take any points $y, y^{\prime} \in Y^{\prime}$ with $\operatorname{dist}\left(y, y^{\prime}\right) \leq \varepsilon$ and let $x=h^{-1}(y)$ and $x^{\prime}=h^{-1}\left(y^{\prime}\right)$. We claim that $\operatorname{dist}\left(x, x^{\prime}\right) \leq \delta+2 R$. By the choice of $\delta$, $\operatorname{dist}\left(g \circ f(x), g \circ f\left(x^{\prime}\right)\right)=\operatorname{dist}\left(g(y), g\left(y^{\prime}\right)\right) \leq \delta=\omega_{g}(\varepsilon)$. Since $\operatorname{dist}\left(g \circ f, \operatorname{id}_{X}\right) \leq$ $R$, we conclude that

$$
\begin{aligned}
\operatorname{dist}\left(x, x^{\prime}\right) & \leq \operatorname{dist}(x, g \circ f(x))+\operatorname{dist}\left(g \circ f(x), g \circ f\left(x^{\prime}\right)\right)+\operatorname{dist}\left(g \circ f\left(x^{\prime}\right), x^{\prime}\right) \\
& \leq R+\operatorname{dist}\left(g(y), g\left(y^{\prime}\right)\right)+R \leq \delta+2 R .
\end{aligned}
$$

Finally, let us show that the set $X^{\prime}$ is large in $X$. Given any point $x \in X$, find a point $x^{\prime} \in X^{\prime}$ with $\operatorname{dist}\left(f(x), f\left(x^{\prime}\right)\right) \leq S$. Then $\operatorname{dist}\left(x, x^{\prime}\right) \leq \operatorname{dist}(x, g \circ f(x))+$ $\operatorname{dist}\left(g \circ f(x), g \circ f\left(x^{\prime}\right)\right)+\operatorname{dist}\left(g \circ f\left(x^{\prime}\right), x^{\prime}\right) \leq R+\omega_{g}(S)+R$ and consequently, $B_{R^{\prime}}\left(X^{\prime}\right)=X$ for $R^{\prime}=2 R+\omega_{g}(S)$. 
(3) $\Rightarrow$ (1) Assume that the spaces $X, Y$ contain bi-uniformly equivalent large subspaces $X^{\prime} \subset X$ and $Y^{\prime} \subset Y$ and let $f: X^{\prime} \rightarrow Y^{\prime}$ be a bi-uniform equivalence. Find $R \in \mathbb{R}$ such that $B_{R}\left(X^{\prime}\right)=X$ and $B_{R}\left(Y^{\prime}\right)=Y$. Take any surjective maps $\varphi: X \rightarrow X^{\prime}$ and $\psi: Y \rightarrow Y^{\prime}$ with $\operatorname{dist}\left(\varphi, \operatorname{id}_{X}\right) \leq R$ and $\operatorname{dist}\left(\psi, \operatorname{id}_{Y}\right) \leq R$. It is easy to see that $\varphi$ and $\psi$ are macro-uniform equivalences and then the composition $\psi^{-1} \circ f \circ \varphi: X \Rightarrow Y$ is a required macro-uniform equivalence between $X$ and $Y$.

\section{3. $\varepsilon$-connected components and uniform multi-maps}

Recall that, for $\varepsilon>0$ and a point $x$ of a metric space $X$, we denote by $C_{\varepsilon}(x)$ the $\varepsilon$-connected component of $x$. This is the set of all points $x^{\prime} \in X$ that can be linked with $x$ by a chain of points $x=x_{0}, x_{1}, \ldots, x_{n}=x^{\prime}$ with $\operatorname{dist}\left(x_{i-1}, x_{i}\right) \leq \varepsilon$ for all $i \leq n$. By $\ell_{\varepsilon}(X)=\left\{C_{\varepsilon}(x) \mid x \in X\right\}$ we denote the family of all $\varepsilon$-connected components of $X$.

Lemma 3.1. Let $\Phi: X \Rightarrow Y$ be a multi-map such that $\Phi^{-1}(Y)=X$. For any real numbers $\delta \geq 0$ and $\varepsilon \geq \omega_{\Phi}(\delta)$, and every point $x \in X$ the image $\Phi\left(C_{\delta}(x)\right)$ lies in the $\varepsilon$-connected component $C_{\varepsilon}(y)$ of any point $y \in \Phi(x)$.

Proof. Given any $x^{\prime} \in C_{\delta}(x)$ and $y^{\prime} \in \Phi(x)$, we need to check that $y^{\prime} \in C_{\varepsilon}(y)$. Find a chain of points $x=x_{0}, x_{1}, \ldots, x_{n}=x^{\prime}$ such that $\operatorname{dist}\left(x_{i-1}, x_{i}\right) \leq \delta$ for all $i \leq n$. Since $X=\Phi^{-1}(Y)$, for every $i \leq n$ we can choose a point $y_{i} \in \Phi\left(x_{i}\right)$ so that $y_{0}=y$ and $y_{n}=y^{\prime}$. It follows from the definition of $\omega_{\Phi}(\delta)$ that for every $i \leq n$, we get

$$
\operatorname{dist}\left(y_{i-1}, y_{i}\right) \leq \operatorname{diam} \Phi\left(\left\{x_{i-1}, x_{i}\right\}\right) \leq \omega_{\Phi}\left(\operatorname{dist}\left(x_{i-1}, x_{i}\right)\right) \leq \omega_{\Phi}(\delta) \leq \varepsilon,
$$

which means that $y=y_{0}, y_{1}, \ldots, y_{n}=y^{\prime}$ is an $\varepsilon$-chain linking the points $y$ and $y^{\prime}$. Consequently, $y^{\prime} \in C_{\varepsilon}(y)$.

Lemma 3.1 will be applied in order to show that some information on the asymptotic properties of the cardinal numbers $\theta_{\delta}^{\varepsilon}(X)$ and $\Theta_{\delta}^{\varepsilon}(X)$ is preserved by bi-uniform equivalences.

Lemma 3.2. Let $\Phi: X \Rightarrow Y$ is a multi-map such that $Y=\Phi(X)$ and $\Phi^{-1}(Y)=X$. For any positive real numbers $\delta<\varepsilon$ and $\delta^{\prime}<\varepsilon^{\prime}$ with $\varepsilon^{\prime} \geq \omega_{\Phi}(\varepsilon), \delta \geq \omega_{\Phi^{-1}}\left(\delta^{\prime}\right)$ we get $\theta_{\delta}^{\varepsilon}(X) \leq \theta_{\delta^{\prime}}^{\varepsilon^{\prime}}(Y)$ and $\Theta_{\delta}^{\varepsilon}(X) \leq \Theta_{\delta^{\prime}}^{\varepsilon^{\prime}}(Y)$.

Proof. For any $\delta$-connected component $C \in \mathcal{C}_{\delta}(X)$ choose a point $y_{C} \in \Phi(C)$. Since $\omega_{\Phi^{-1}}\left(\delta^{\prime}\right) \leq \delta$, we can apply Lemma 3.1 to prove that for any distinct components $C, C^{\prime} \in C_{\delta}(X)$ the points $y_{C}$ and $y_{C}^{\prime}$ lie in distinct $\delta^{\prime}$-components of $Y$. 
Therefore the map

$$
\varphi: \mathscr{C}_{\delta}(X) \rightarrow \mathscr{C}_{\delta^{\prime}}(Y), \quad \varphi: C \mapsto C_{\delta^{\prime}}\left(y_{C}\right),
$$

is injective.

By Lemma 3.1, for any point $x \in X$ the set $\Phi\left(C_{\varepsilon}(x)\right)$ lies in $C_{\varepsilon^{\prime}}(y)$ for any $y \in \Phi(x)$. Now the injectivity of the map $\varphi$ implies that

$$
\left|C_{\varepsilon}(x) / \mathscr{C}_{\delta}(X)\right| \leq\left|C_{\varepsilon^{\prime}}(y) / \mathscr{C}_{\delta^{\prime}}(Y)\right| \leq \Theta_{\delta^{\prime}}^{\varepsilon^{\prime}}(Y)
$$

and hence $\Theta_{\delta}^{\varepsilon}(X) \leq \Theta_{\delta^{\prime}}^{\varepsilon^{\prime}}(Y)$.

Next, find a point $y \in Y$ with $\theta_{\delta^{\prime}}^{\varepsilon^{\prime}}(Y)=\left|C_{\varepsilon^{\prime}}(y) / \complement_{\delta^{\prime}}(Y)\right|$ and choose any point $x \in \Phi^{-1}(y)$. Then

$$
\theta_{\delta}^{\varepsilon}(X) \leq\left|C_{\varepsilon}(x) / \mathscr{C}_{\delta}(X)\right| \leq\left|C_{\varepsilon^{\prime}}(y) / \mathscr{C}_{\delta^{\prime}}(Y)\right|=\theta_{\delta^{\prime}}^{\varepsilon^{\prime}}(Y) .
$$

\section{Towers}

The Characterization Theorems announced in the introduction will be proved by induction on partially ordered sets called towers. A typical example of a tower is the set $\left\{B_{2^{n}}(x) \mid x \in X, n \in \mathbb{Z}\right\}$ of closed $2^{n}$-balls of an ultrametric space $X$, ordered by the inclusion relation. To give a precise definition of a tower we need to recall some standard notions related to partially ordered sets.

4.1. Partially ordered sets. A partially ordered set is a set $T$ endowed with a reflexive antisymmetric transitive relation $\leq$.

A partially ordered set $T$ is called $\uparrow$-directed (resp. $\downarrow$-directed) if for any two points $x, y \in T$ there is a point $z \in T$ such that $z \geq x$ and $z \geq y$ (resp. $z \leq x$ and $z \leq y)$.

A subset $C$ of a partially ordered set $T$ is called $\downarrow$-cofinal (resp. $\uparrow$-cofinal) if for every $x \in T$ there is $y \in C$ such that $y \leq x$ (resp. $y \geq x$ ). A subset $C \subset T$ is called $\uparrow$-cofinal in $T$ if $C$ is $\downarrow$-cofinal and $\uparrow$-cofinal in $T$.

By the lower cone (resp. upper cone) of a point $x \in T$ we understand the set $\downarrow x=\{y \in T \mid y \leq x\}$ (resp. $\uparrow x=\{y \in T \mid y \geq x\}$ ). A subset $A \subset T$ will be called a lower (resp. upper) set if $\downarrow a \subset A$ (resp. $\uparrow a \subset A$ ) for all $a \in A$. For two points $x \leq y$ of $T$ the intersection $[x, y]=\uparrow x \cap \downarrow y$ is called the order interval with end-points $x, y$.

A partially ordered set $T$ is a tree if $T$ is $\downarrow$-directed and for each point $x \in T$ the lower cone $\downarrow x$ is well-ordered (in the sense that each subset $A \subset \downarrow x$ has the smallest element). 
4.2. Introducing towers. A partially ordered set $T$ is called a tower if $T$ is $\uparrow$ directed and for every points $x \leq y$ in $T$ the order interval $[x, y] \subset T$ is finite and linearly ordered.

This definition implies that for every point $x$ in a tower $T$ the upper set $\uparrow x$ is linearly ordered and is order isomorphic to a subset of $\omega$. Since $T$ is $\uparrow$-directed, for any points $x, y \in T$ the upper sets $\uparrow x$ and $\uparrow y$ have non-empty intersection and this intersection has the smallest element $x \wedge y=\min (\uparrow x \cap \uparrow y)$ (because each order interval in $X$ is finite). Thus, any two points $x, y$ in a tower have the smallest upper bound $x \wedge y$.

It follows that for each point $x \in T$ of a tower the lower cone $\downarrow x$ endowed with the reverse partial order is a tree of at most countable height.

4.3. Levels of a tower. The definition of a tower $T$ includes the condition that for any points $x \leq y$ of $T$ the order interval $[x, y]=\uparrow x \cap \downarrow y$ is linearly ordered and finite. This allows us to define levels of the tower $T$ as follows.

Given two points $x, y \in T$ we write $\operatorname{lev}_{T}(x) \leq \operatorname{lev}_{T}(y)$ if

$$
|[x, x \wedge y]| \geq|[y, x \wedge y]|
$$

Also we $\operatorname{write} \operatorname{lev}_{T}(x)=\operatorname{lev}_{T}(y)$ if $|[x, x \wedge y]|=|[y, x \wedge y]|$.

The relation

$$
\left\{(x, y) \in T \times T \mid \operatorname{lev}_{T}(x)=\operatorname{lev}_{T}(y)\right\}
$$

is an equivalence relation on $T$ dividing the tower $T$ into equivalence classes called

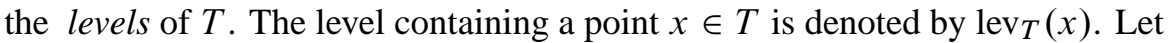

$$
\operatorname{Lev}(T)=\left\{\operatorname{lev}_{T}(x) \mid x \in T\right\}
$$

denote the set of levels of $T$ and

$$
\operatorname{lev}_{T}: T \rightarrow \operatorname{Lev}(T), \quad \operatorname{lev}_{T}: x \mapsto \operatorname{lev}_{T}(x)
$$

stand for the quotient map called the level map. If the tower $T$ is clear from the context, we omit the subscript $T$ and write lev instead of $\operatorname{lev}_{T}$.

The set $\operatorname{Lev}(T)$ of levels of $T$ endowed with the $\operatorname{order} \operatorname{lev}_{T}(x) \leq \operatorname{lev}_{T}(y)$ is a linearly ordered set, order isomorphic to a subset of integers. For a level $\lambda \in \operatorname{Lev}(T)$ by $\lambda+1$ (resp. $\lambda-1$ ) we denote the successor (resp. the predecessor) of $\lambda$ in the level set $\operatorname{Lev}(T)$. If $\lambda$ is a maximal (resp. minimal) level of $T$, then we put $\lambda+1=\varnothing$ (resp. $\lambda-1=\emptyset$ ).

An embedding of the level set $\operatorname{Lev}(T)$ into $\mathbb{Z}$ can be constructed as follows. Pick any point $\theta \in T$ and consider the map $e_{\theta}: \operatorname{Lev}(T) \rightarrow \mathbb{Z}$ assigning to each level $\operatorname{lev}_{T}(x) \in \operatorname{Lev}(T)$ the integer number

$$
|[x, x \wedge \theta]|-|[\theta, x \wedge \theta]|
$$


In such a way we label the levels of $T$ by integer numbers so that the point $\theta$ sits on the zeros level.

The following model of the famous Eiffel tower is an example of a tower having seven levels.

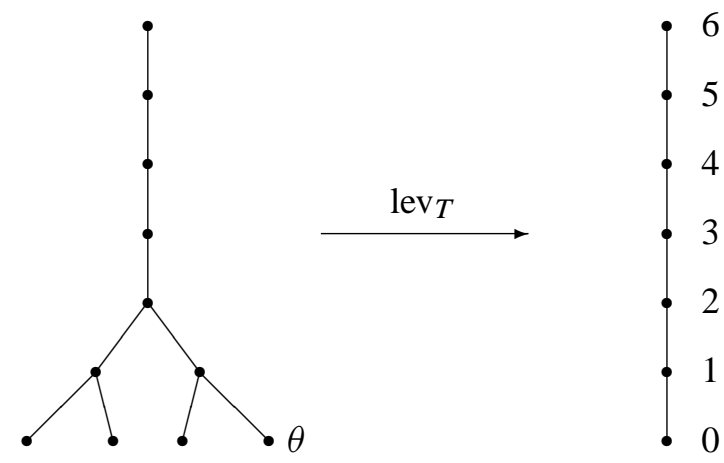

A tower $T$ is called $\downarrow$-bounded (resp. $\uparrow$-bounded) if the level set $\operatorname{Lev}(T)$ has the smallest (resp. largest) element. Otherwise $T$ is called $\downarrow$-unbounded (resp. $\uparrow$ unbounded). A tower $T$ is called $\uparrow$-unbounded it it is $\downarrow$-unbounded and $\uparrow$-unbounded. Let us observe that $\uparrow$-bounded towers endowed with the reverse partial order are trees of at most countable height.

4.4. A tower induced by a decomposition of a group. Let $G$ be a group written as the countable union $G=\bigcup_{n \in \omega} G_{n}$ of a strictly increasing sequence

$$
\{e\}=G_{0} \subset G_{1} \subset \cdots
$$

of subgroups of $G$.

Consider the family of cosets $T=\left\{x G_{n} \mid x \in G, n \in \omega\right\}$ partially ordered by the inclusion relation. It is easy to check that the partially order set $T$ is a tower. This tower is $\downarrow$-bounded and $\uparrow$-unbounded. For every $n \in \omega$ the family of cosets $\left\{x G_{n} \mid x \in G\right\}$ forms a level of $T$. The minimal level of $G$ consists of the singletons and hence can be identified with the whole group $G$.

4.5. The boundary of a tower. By a branch of a tower $T$ we understand a maximal linearly ordered subset of $T$. The family of all branches of $T$ is denoted by $\partial T$ and is called the boundary of $T$. The boundary $\partial T$ carries an ultrametric that can be defined as follows.

Let $f: \operatorname{Lev}(T) \rightarrow[0, \infty)$ be a strictly increasing function such that

- $\inf f(\operatorname{Lev}(T))=0$ if $T$ is $\downarrow$-unbounded, and

- $\sup f(\operatorname{Lev}(T))=\infty$ if $T$ is $\uparrow$-unbounded. 
Such a map $f$ will be called a scaling function on $\operatorname{Lev}(T)$.

Given two branches $x, y \in \partial T$ let

$$
\rho_{f}(x, y)= \begin{cases}0 & \text { if } x=y, \\ f\left(\operatorname{lev}_{T}(\min x \cap y)\right) & \text { if } x \neq y .\end{cases}
$$

It is a standard exercise to check that $\rho_{f}$ is a well-defined ultrametric on the boundary $\partial T$ of $T$ turning $\partial T$ into a complete ultrametric space. The following easy proposition says that the bi-uniform structure on $\partial T$ induced by the ultrametric $\rho_{f}$ does not depend on the choice of a scaling function $f$.

Proposition 4.1. For any two scaling functions $f, g: \operatorname{Lev}(T) \rightarrow(0, \infty)$ the identity map id: $\left(\partial T, \rho_{f}\right) \rightarrow\left(\partial T, \rho_{g}\right)$ is a bi-uniform equivalence.

In the sequel we assume that the boundary $\partial T$ of any tower $T$ is endowed with the ultrametric $\rho_{f}$ induced by some scaling function $f: \operatorname{Lev}(T) \rightarrow(0, \infty)$.

4.6. Degrees of points of a tower. For a point $x \in T$ and a level $\lambda \in \operatorname{Lev}(T)$ let $\operatorname{pred}_{\lambda}(x)=\lambda \cap \downarrow x$ be the set of predecessors of $x$ on the $\lambda$-th level and $\operatorname{deg}_{\lambda}(x)=$ $\left|\operatorname{pred}_{\lambda}(x)\right|$. For $\lambda=\operatorname{lev}_{T}(x)-1$, the set $\operatorname{pred}_{\lambda}(x)$, called the set of parents of $x$, is denoted by $\operatorname{pred}(x)$. The cardinality $|\operatorname{pred}(x)|$ is called the degree of $x$ and is denoted by $\operatorname{deg}(x)$. Thus, $\operatorname{deg}(x)=\operatorname{deg}_{\operatorname{lev}_{T}(x)-1}(x)$. It follows that $\operatorname{deg}(x)=0$ if and only if $x$ is a minimal element of $T$.

For levels $\lambda, l \in \operatorname{Lev}(T)$ let

$$
\operatorname{deg}_{\lambda}^{l}(T)=\min \left\{\operatorname{deg}_{\lambda}(x) \mid \operatorname{lev}_{T}(x)=l\right\}
$$

and

$$
\operatorname{Deg}_{\lambda}^{l}(T)=\sup \left\{\operatorname{deg}_{\lambda}(x) \mid \operatorname{lev}_{T}(x)=l\right\} .
$$

Now let us introduce several notions related to degrees. We define a tower $T$ to be

- homogeneous if $\operatorname{deg}_{\lambda}^{\ell}(T)=\operatorname{Deg}_{\lambda}^{\ell}(T)$ for any level $\lambda \leq \ell$ of $T$;

- pruned if $\operatorname{deg}_{\lambda}^{\lambda+1}(T)>0$ for every non-maximal level $\lambda$ of $T$;

- $\uparrow$-branching if for all $\lambda \in \operatorname{Lev}(T)$ there exists $l \in \operatorname{Lev}(T)$ with $\operatorname{Deg}_{\lambda}^{l}(T)>1$;

- $\downarrow$-branching if for all $\lambda \in \operatorname{Lev}(T)$ there exists $l \in \operatorname{Lev}(T)$ with $\operatorname{deg}_{l}^{\lambda}(T)>1$;

- $\uparrow$-branching if $T$ is both $\downarrow$-branching and $\uparrow$-branching.

It is easy to check that a tower $T$ is pruned if and only if each branch of $T$ meets each level of $T$. A tower $T$ is $\uparrow$-branching if no level $\lambda \in \operatorname{Lev}(T)$ has an upper bound in $T$.

By a binary tower we understand an $\uparrow$-unbounded homogeneous tower $T$ such that $\operatorname{deg}_{\lambda}^{\lambda+1}(T)=2$ for each non-maximal level $\lambda$ of $T$. It is clear that each binary tower is pruned and $\uparrow$-branching. 
Remark 4.2. The Cantor bi-cube $2^{<\mathbb{Z}}$ (resp. Cantor macro-cube $2^{<\mathbb{N}}$ ) can be identified with the boundary $\partial T_{2}$ of a $\downarrow$-unbounded (resp. $\downarrow$-bounded) binary tower $T_{2}$.

There is a direct dependence between the degrees of points of the tower $T$ and the capacities of the balls in the ultrametric space $\partial T$. We recall that for positive real numbers $\delta \leq \varepsilon$ and a point $x \in X$ by $\left|C_{\varepsilon}(x) / \mathscr{C}_{\delta}(X)\right|$ we denote the cardinality of the set $\left\{C_{\delta}(y) \mid y \in C_{\varepsilon}(x)\right\}$ of $\delta$-connected components of $X$ that lie in the $\varepsilon$-connected component of $y$ in $X$. If $X$ is an ultrametric space then $C_{\varepsilon}(x) / \mathcal{C}_{\delta}(X)$ is equal to the number of $\delta$-balls composing the $\varepsilon$-ball $B_{\varepsilon}(x)$.

Proposition 4.3. Let be a tower and $f: \operatorname{Lev}(T) \rightarrow(0, \infty)$ be a scaling function determining the ultrametric $\rho_{f}$ on the boundary $\partial T$ of $T$. For any branch $\beta \in \partial T$, a point $x \in \beta$ with $n=\operatorname{lev}_{T}(x)$, and a level $k \leq n$ of $T$ we get $\operatorname{deg}_{k}(x)=$ $\left|C_{f(n)}(\beta) / \complement_{f(k)}(\partial T)\right|$. Consequently,

$$
\operatorname{deg}_{k}^{n}(T)=\theta_{f(k)}^{f(n)}(\partial T) \quad \text { and } \quad \operatorname{Deg}_{k}^{n}(T)=\Theta_{f(k)}^{f(n)}(\partial T) .
$$

The proof is easy and is left to the reader as an exercise.

4.7. Assigning a tower to a metric space. In the preceding section to each tower $T$ we have assigned the ultrametric space $\partial T$. In this section we describe the converse operation assigning to each metric space $X$ a pruned tower $T_{X}^{L}$ whose boundary $\partial T_{X}^{L}$ is canonically related to the space $X$.

A subset $L \subset[0, \infty)$ is called a level set if

- $\sup L=\infty$ and hence $L$ is $\uparrow$-cofinal in $[0, \infty)$;

- $L$ is a tower in the sense that $[x, y] \cap L$ is finite for all $x, y \in L$;

- inf $L=0$ if $L \cap(-\infty, x]$ is infinite for some $x \in L$.

A level set $L \subset[0, \infty)$ is called $\downarrow$-bounded if it has the smallest element. Otherwise, $L$ is $\downarrow$-unbounded.

Given a metric space $X$ and a level set $L \subset[0, \infty)$ consider the set

$$
T_{X}^{L}=\left\{\left(C_{\lambda}(x), \lambda\right) \mid x \in X, \lambda \in L\right\}
$$

endowed with the partial order $\left(C_{\lambda}(x), \lambda\right) \leq\left(C_{l}(y), l\right)$ if $\lambda \leq l$ and $C_{\lambda}(x) \subset C_{l}(y)$. Here, as expected, $C_{\lambda}(x)$ stands for the $\lambda$-connected component of $x$ in $X$.

Proposition 4.4. The partially ordered set $T_{X}^{L}$ is a pruned tower whose level set $\operatorname{Lev}\left(T_{X}^{L}\right)$ can be identified with $L$. If the metric space $X$ is isometrically homogeneous, then the tower $T_{X}^{L}$ is homogeneous.

Proof. To detect that the partially ordered set $T_{X}^{L}$ is $\uparrow$-directed, let us take two elements $\left(C_{\alpha}(x), \alpha\right),\left(C_{\beta}(y), \beta\right) \in T_{X}^{L}$ and look for a number $\lambda \in L$ such that 
$\lambda \geq \max \{\alpha, \beta, \operatorname{dist}(x, y)\}$ (such a number $\lambda$ exists because $\sup L=\infty$ ). Then $\left(C_{\lambda}(x), \lambda\right)$ is an upper bound for $\left(C_{\alpha}(x), \alpha\right)$ and $\left(C_{\beta}(y), \beta\right)$ in $T_{X}^{L}$.

Next, given two points $u=\left(C_{\alpha}(x), \alpha\right), v=\left(C_{\beta}(y), \beta\right)$ in $T_{X}^{L}$ with $u \leq v$, we need to check that the order interval $[u, v]$ is linearly ordered and finite. Take any two points $t_{1}, t_{2} \in[u, v]$ and for every $i \in\{1,2\}$ find a point $z_{i} \in X$ and a real number $\lambda_{i} \in L$ such that $t_{i}=\left(C_{\lambda_{i}}\left(z_{i}\right), \lambda_{i}\right)$. It follows from $u \leq t_{i} \leq v$ that $\alpha \leq \lambda_{i} \leq \beta$ and $C_{\alpha}(x) \subset C_{\lambda_{i}}\left(z_{i}\right) \subset C_{\beta}(y)$.

Without loss of generality, we may assume that $\lambda_{1} \leq \lambda_{2}$. Since $C_{\alpha}(x) \subset$ $C_{\lambda_{2}}\left(z_{1}\right) \cap C_{\lambda_{2}}\left(z_{2}\right)$, the $\lambda_{2}$-connected components $C_{\lambda_{2}}\left(z_{1}\right), C_{\lambda_{2}}\left(z_{2}\right)$ coincide and hence $C_{\lambda_{1}}\left(z_{1}\right) \subset C_{\lambda_{2}}\left(z_{1}\right)=C_{\lambda_{2}}\left(z_{2}\right)$. Thus, $t_{1} \leq t_{2}$, showing that $[u, v]$ is linearly ordered.

By the same reason, $\lambda_{1}=\lambda_{2}$ implies $t_{1}=t_{2}$, ensuring that the projection

$$
\operatorname{pr}:[u, v] \rightarrow[\alpha, \beta] \cap L, \quad \operatorname{pr}:\left(C_{\lambda}(z), \lambda\right) \mapsto \lambda,
$$

is bijective and $|[u, v]| \leq|[\alpha, \beta] \cap L|$ is finite.

It follows that the projection

$$
\operatorname{pr}: T_{X}^{L} \rightarrow L, \quad \operatorname{pr}:\left(C_{\lambda}(x), \lambda\right) \mapsto \lambda,
$$

is a monotone surjective level-preserving map and for every $\lambda \in L$ the preimage $\operatorname{pr}^{-1}(\lambda)=\left\{\left(C_{\lambda}(x), \lambda\right) \mid x \in X\right\}$ coincides with a level of the tower $T_{X}^{L}$. So, the set $L$ can be identified with the set $\operatorname{Lev}\left(T_{X}^{L}\right)$ of levels of the tower $T_{X}^{L}$.

To see that the tower $T_{X}^{L}$ is pruned, take any point $t=\left(C_{\lambda}(x), \lambda\right) \in T_{X}$ on a non-minimal level $\lambda \in L$ and let $\lambda^{-} \in L$ be the predecessor of $\lambda$ in $L$. Then the element $\left(C_{\lambda^{-}}(x), \lambda^{-}\right)$is a parent of $t$, showing that $\operatorname{deg}(t)>0$ and $T_{X}$ is pruned.

If the metric space $X$ is isometrically homogeneous, then the tower $T_{X}^{L}$ is homogeneous because for each point $t=\left(C_{\lambda}(x), \lambda\right) \in T_{X}^{L}$ and each level $\ell \in L$, $\ell \leq \lambda$, the degree $\operatorname{deg}_{\ell}(t)=\left|C_{\lambda}(x) / \mathscr{C}_{\ell}(X)\right|$ does not depend on the point $x$. So, $\operatorname{deg}_{\ell}^{\lambda}\left(T_{X}^{L}\right)=\operatorname{Deg}_{\ell}^{\lambda}\left(T_{X}^{L}\right)$, showing that the tower $T_{X}^{L}$ is homogenous.

The tower $T_{X}^{L}$ is called the canonical $L$-tower of a metric space $X$. The boundary $\partial T_{X}^{L}$ is endowed with the ultrametric $\rho_{\text {id }}$ induced by the identity scaling function id: $L \rightarrow[0, \infty)$. This ultrametric on $\partial T_{X}^{L}$ will be called canonical.

Observe that for each point $x \in X$ the set $C_{L}(x)=\left\{\left(C_{\lambda}(x), \lambda\right) \mid \lambda \in L\right\}$ is a branch of the tower, so the map

$$
C_{L}: X \rightarrow \partial T_{X}^{L}, \quad C_{L}: x \mapsto C_{L}(x),
$$

called the canonical map, is well defined.

Proposition 4.5. (1) $\operatorname{dist}\left(C_{L}(x), C_{L}(y)\right) \leq \inf \{\lambda \in L \mid \lambda \geq d(x, y)\}$ for all $x, y \in X$.

(2) The canonical map $C_{L}: X \rightarrow \partial T_{X}^{L}$ is macro-uniform.

(3) If $0 \notin L$, then the canonical map $C_{L}$ is micro-uniform. 
(4) If $L$ is $\downarrow$-bounded, then the canonical map $C_{L}$ is surjective.

(5) The canonical map $C_{L}$ has dense image $C_{L}(X)$ in $\partial T_{X}^{L}$.

(6) The inverse multi-map $C_{L}^{-1}: \partial T_{X}^{L} \Rightarrow X$ is macro-uniform if and only if $X$ has macro-uniform dimension zero.

(7) If $L$ is $\downarrow$-unbounded, then the inverse multi-map $C_{L}^{-1}: \partial T_{X}^{L} \Rightarrow X$ is microuniform if and only if $X$ has micro-uniform dimension zero.

Proof. (1) Given any two points $x, y \in X$ let $\lambda=\inf (L \cap[\operatorname{dist}(x, y), \infty))$ and observe that $C_{\lambda}(x)=C_{\lambda}(y)$, which implies that $\operatorname{dist}\left(C_{L}(x), C_{L}(y)\right) \leq \lambda$.

(2) The preceding item implies immediately that the canonical map $C_{L}: X \rightarrow$ $\partial T_{X}^{L}$ is macro-uniform.

(3) Assume that $0 \notin L$. If $\inf L>0$, then for any positive $\delta<\inf L$ we get $\omega_{C_{L}}(\delta)=0$ and thus $C_{L}$ is micro-uniform.

If inf $L=0$, then for every $\varepsilon>0$ we can find $\delta \in L \cap(0, \varepsilon]$ and observe that $\omega_{C_{L}}(\delta)=\delta \leq \varepsilon$, showing that $C_{L}$ is micro-uniform.

(4) If $L$ is $\downarrow$-bounded, then $L$ has a minimal element $\lambda_{0}$. It follows that each branch $\beta$ of the tower $T_{X}^{L}$ is equal to $C_{L}(x)$ for a point $x \in X$ whose $\lambda_{0}$-connected component $C_{\lambda_{0}}(x)$ coincides with the smallest element of the branch $\beta$. In this case the map $C_{L}$ is surjective.

(5) If $L$ is $\downarrow$-bounded, then the map $C_{L}$ is surjective by the preceding item and hence has dense image $C_{L}(X)$ in $\partial T_{X}^{L}$.

If $L$ is $\downarrow$-unbounded, then inf $L=0 \notin L$. Given any branch $\beta \in \partial T_{X}^{L}$ and any $\varepsilon>0$, we can find $\lambda \in L \cap(0, \varepsilon)$ and a point $x \in X$ with $\left(C_{\lambda}(x), \lambda\right) \in \beta$. Then $\operatorname{dist}\left(\beta, C_{L}(x)\right) \leq \lambda<\varepsilon$, showing that the image $C_{L}(X)$ is dense in $\partial T_{X}^{L}$.

(6) Assume that the inverse multi-map $C_{L}^{-1}: \partial T_{X}^{L} \Rightarrow X$ is macro-uniform. To show that $X$ has macro-uniform dimension zero, we need to show that mesh $\bigodot_{\delta}(X)$ is finite for every $\delta<\infty$. Find any $\lambda \in L \cap[\delta, \infty)$ and put $\varepsilon=\omega_{C_{L}^{-1}}(\lambda)$.

We claim that mesh $\digamma_{\delta}(X) \leq \varepsilon$. Indeed, given any $\delta$-connected component $C \in \mathcal{C}_{\delta}(X)$ and any points $x, y \in C$ we get dist $\left(C_{L}(x), C_{L}(y)\right) \leq \lambda$ and $\operatorname{dist}(x, y) \leq$ $\operatorname{diam} C_{L}^{-1}\left(\left\{C_{L}(x), C_{L}(y)\right\}\right) \leq \omega_{C_{L}^{-1}}(\lambda) \leq \varepsilon$. Then $\operatorname{diam} C \leq \varepsilon$ and mesh $C_{\delta}(X) \leq$ $\varepsilon$, showing that the metric space $X$ has macro-uniform dimension zero.

Now assume conversely that $X$ has macro-uniform dimension zero. In order to show that the inverse multi-map $C_{L}^{-1}: \partial T_{X}^{L} \Rightarrow X$ is macro-uniform, given any $\delta<\infty$ find $\lambda \in L \cap[\delta, \infty)$ and put $\varepsilon=$ mesh $\mathscr{C}_{\lambda}(X)$. The number $\varepsilon$ is finite because $X$ has macro-uniform dimension zero. We claim that $\omega_{C_{L}^{-1}}(\delta) \leq \varepsilon$. Take any subset $A \subset \partial T_{X}^{L}$ with $\operatorname{diam} A \leq \delta$. We need to show that $\operatorname{diam} C_{L}^{-1}(A) \leq \varepsilon$. Take any points $x, y \in C_{L}^{-1}(A)$ and observe that $C_{L}(x), C_{L}(y) \in A$. Since $\operatorname{dist}\left(C_{L}(x), C_{L}(y)\right) \leq$ $\delta \leq \lambda, C_{\lambda}(x)=C_{\lambda}(y)$ and then $\operatorname{dist}(x, y) \leq \operatorname{mesh} \mathscr{C}_{\lambda}(X)=\varepsilon$ and hence $\operatorname{diam} A \leq \varepsilon$.

(7) Assume that $L$ is $\downarrow$-unbounded. If $X$ has micro-uniform dimension zero, then for any $\varepsilon>0$ we can find $\lambda \in L \cap(0, \varepsilon)$ and take $\delta>0$ so small that mesh $\bigodot_{\delta}(X) \leq \lambda$. Repeating the argument from the preceding item, we can prove that ${ }^{{ }^{-1}} C_{L}^{-1}(\delta) \leq \lambda \leq \varepsilon$, 
showing that $C_{L}^{-1}$ is micro-uniform.

Finally assume that $C_{L}^{-1}$ is micro-uniform. Then for every $\varepsilon>0$ we can find $\delta \in L$ with $\omega_{C_{L}^{-1}}(\delta) \leq \varepsilon$. Repeating the argument from the proof of the preceding item, we can check that mesh $\varrho_{\delta}(X) \leq \varepsilon$, showing that $X$ has micro-uniform dimension zero.

The statements (2), (3), (6), (7) of Proposition 4.5 imply:

Corollary 4.6. Let $L \subset[0, \infty)$ be a level set. The canonical map $C_{L}: X \rightarrow \partial T_{X}^{L}$ of a metric space $X$ into the boundary of its canonical L-tower $T_{X}^{L}$ is:

(1) a macro-uniform embedding if and only if $X$ has macro-uniform dimension zero;

(2) a micro-uniform embedding (if and) only if $X$ has micro-uniform dimension zero (and $L$ is $\downarrow$-unbounded);

(3) a bi-uniform embedding (if and) only if $X$ has bi-uniform dimension zero (and $L$ is $\downarrow$-unbounded).

Combining this corollary with Proposition 4.5 (4), (5) we get another

Corollary 4.7. Let $L \subset[0, \infty)$ be a level set. The canonical map $C_{L}: X \rightarrow \partial T_{X}^{L}$ of a metric space $X$ into the boundary of its canonical L-tower is:

(1) a macro-uniform equivalence (if and) only if $X$ has macro-uniform dimension zero (and $L$ is $\downarrow$-bounded);

(2) a micro-uniform equivalence (if and) only if $X$ is a complete metric space of micro-uniform dimension zero (and $L$ is $\downarrow$-unbounded);

(3) a bi-uniform equivalence (if and) only if $X$ is a complete metric space of biuniform dimension zero (and $L$ is $\downarrow$-unbounded).

Proof. (1) The first item is a direct consequence of Corollary 4.6(1) and Proposition 4.5(4).

(2) If $C_{L}: X \rightarrow \partial T_{X}^{L}$ is a micro-uniform equivalence (that is, a uniform homeomorphism), then the metric space $X$ is complete because so is the ultrametric space $\partial T_{X}^{L}$. Corollary 4.6 (2) implies that $X$ has micro-uniform dimension zero.

Now assume conversely that the metric space $X$ is complete and has microuniform dimension zero and the level set $L$ is $\downarrow$-unbounded. By Corollary 4.6 (2), the canonical map $C_{L}: X \rightarrow \partial T_{X}^{L}$ is a micro-uniform embedding and by Proposition 4.5(5), the image $C_{L}(X)$ is dense in $\partial T_{X}^{L}$. The metric space $C_{L}(X) \subset \partial T_{X}^{L}$, being uniformly homeomorphic to the complete metric space $X$, is complete and hence coincides with $\partial T_{X}^{L}$. Then the canonical map $C_{L}$, being a surjective microuniform embedding, is a micro-uniform equivalence.

(3) The third statement can be proved by analogy with the second one. 
Remark 4.8. The correspondence between towers and metric spaces discussed in this section resembles in spirit the correspondence between $\mathbb{R}$-trees and ultrametric spaces discussed in [Hug], [MPM].

\section{Tower morphisms}

In this section we discuss morphisms between towers.

5.1. Introducing tower morphisms. In this subsection we introduce several kinds of morphisms between towers $S, T$.

A map $\varphi: S \rightarrow T$ is defined to be

- monotone if for any $x, y \in S$ the inequality $x<y$ implies $\varphi(x)<\varphi(y)$;

- level-preserving if there is an injective map $\varphi_{\mathrm{Lev}}: \operatorname{Lev}(S) \rightarrow \operatorname{Lev}(T)$ making diagram

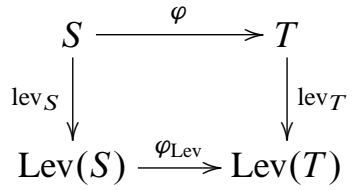

commutative.

If $\varphi: S \rightarrow T$ is a monotone level-preserving map, then the induced map $\varphi_{\mathrm{Lev}}: \operatorname{Lev}(S) \rightarrow \operatorname{Lev}(T)$ is monotone and injective.

A monotone level-preserving map $\varphi: S \rightarrow T$ is called

- a tower isomorphism if it is bijective;

- a tower embedding if it is injective;

- a tower immersion if it is almost injective in the sense that for any points $x, x^{\prime} \in S$ with $\varphi(x)=\varphi\left(x^{\prime}\right)$ we get $\operatorname{lev}_{S}\left(x \wedge x^{\prime}\right) \leq \max \left\{\operatorname{lev}_{S}(x), \operatorname{lev}_{S}\left(x^{\prime}\right)\right\}+1$.

Proposition 5.1. If $\varphi: S \rightarrow T$ is a tower embedding, then for any $x, x^{\prime} \in S$ the inequality $x<x^{\prime}$ is equivalent to $\varphi\left(x^{\prime}\right)<\varphi\left(x^{\prime}\right)$.

Proof. If $x<x^{\prime}$, then $\varphi(x)<\varphi\left(x^{\prime}\right)$ by the monotonicity of $\varphi$.

Now assume that $\varphi(x)<\varphi\left(x^{\prime}\right)$. The chain of the inequalities $\varphi(x) \leq \varphi\left(x^{\prime}\right) \leq$ $\varphi\left(x \wedge x^{\prime}\right)$ and the level-preserving property of $\varphi$ imply that $\operatorname{lev}(x) \leq \operatorname{lev}\left(x^{\prime}\right) \leq \operatorname{lev}(x \wedge$ $\left.x^{\prime}\right)$. Then there is a point $x^{\prime \prime} \in\left[x, x \wedge x^{\prime}\right]$ with $\operatorname{lev}\left(x^{\prime \prime}\right)=\operatorname{lev}\left(x^{\prime}\right)$. For this point $x^{\prime \prime}$ we get $\varphi(x) \leq \varphi\left(x^{\prime \prime}\right) \leq \varphi\left(x \wedge x^{\prime}\right)$. Taking into account that $\operatorname{lev}\left(\varphi\left(x^{\prime \prime}\right)\right)=\operatorname{lev}\left(\varphi\left(x^{\prime}\right)\right)$ and the order interval $\left[\varphi(x), \varphi\left(x \wedge x^{\prime}\right)\right] \subset T$ is linearly ordered, we conclude that $\varphi\left(x^{\prime \prime}\right)=\varphi\left(x^{\prime}\right)$ and $x^{\prime \prime}=x^{\prime}$ by the injectivity of $\varphi$. Then $x \leq x^{\prime \prime}=x^{\prime}$ and $\varphi(x) \neq \varphi\left(x^{\prime}\right)$ implies $x<x^{\prime}$. 
5.2. Induced multi-maps between boundaries of towers. Each monotone map $\varphi: S \rightarrow T$ between towers induces a multi-map $\partial \varphi: \partial S \Rightarrow \partial T$ assigning to a branch $\beta \subset S$ the set $\partial \varphi(\beta) \subset \partial T$ of all branches of $T$ that contain the linearly ordered subset $\varphi(\beta)$ of $T$. It follows that $\partial \varphi(\beta) \neq \emptyset$ and hence $(\partial \varphi)^{-1}(\partial T)=\partial S$.

The following proposition describes some properties of the boundary multi-maps.

Proposition 5.2. For a monotone map $\varphi: S \rightarrow T$ defined on a pruned tower $S$ the induced multi-map $\partial \varphi: \partial S \Rightarrow \partial T$ is

(1) single valued if for all $\beta \in \partial S$ and for all $\lambda \in \operatorname{Lev}(T)$ there exists $x \in \beta$ with $\operatorname{lev}_{T}(\varphi(x)) \leq \lambda$

(2) micro-uniform if for all $\lambda \in \operatorname{Lev}(T)$ there exists $v \in \operatorname{Lev}(S)$ such that $\operatorname{lev}_{S}(x) \leq v \Rightarrow \operatorname{lev}_{T}(\varphi(x)) \leq \lambda$ for all $x \in S$

(3) macro-uniform if for all $v \in \operatorname{Lev}(S)$ there exists $\lambda \in \operatorname{Lev}(T)$ such that $\operatorname{lev}_{S}(x) \leq v \Rightarrow \operatorname{lev}_{T}(\varphi(x)) \leq \lambda$ for all $x \in S$.

Proof. We recall that the boundaries $\partial S$ and $\partial T$ are endowed with ultrametrics $\rho_{f}$ and $\rho_{g}$ generated by some scaling functions $f: \operatorname{Lev}(S) \rightarrow[0, \infty)$ and $g: \operatorname{Lev}(T) \rightarrow$ $[0, \infty)$.

(1) Assuming that $\partial \varphi$ is not single-valued, we can find a branch $\beta \in \partial S$ and two distinct branches $b_{1}, b_{2} \in \partial T$ such that $b_{1} \cap b_{2} \supset \varphi(\beta)$. Since $b_{1} \neq b_{2}$, there is a level $\lambda \in \operatorname{Lev}(T)$ of $T$ such that the intersections $b_{1} \cap \lambda$ and $b_{2} \cap \lambda$ are not empty and distinct. For this level $\lambda$ no point $x \in \beta$ exists with $\operatorname{lev}_{T}(\varphi(x)) \leq \lambda$.

(2) Assume that for all $\lambda \in \operatorname{Lev}(T)$ there exists $v \in \operatorname{Lev}(S)$ such that $\operatorname{lev}_{S}(x) \leq v \Rightarrow \operatorname{lev}_{T}(\varphi(x)) \leq \lambda$ for all $x \in S$. The micro-uniform property of the boundary map $\partial \varphi: \partial S \Rightarrow \partial T$ follows as soon as for every $\varepsilon>0$ we find $\delta>0$ with $\omega_{\partial \varphi}(\delta) \leq \varepsilon$.

If the tower $T$ is $\downarrow$-bounded, then the set $\operatorname{Lev}(T)$ has the smallest element $\lambda_{0}$. By our assumption, for the level $\lambda_{0}$ there is a level $v \in \operatorname{Lev}(S)$ such that $\operatorname{lev}_{T}(\varphi(x)) \leq \lambda_{0}$ for all $x \in S$ with $\operatorname{lev}_{S}(x) \leq v$. Let $\delta=f(v)$. We claim that $\omega_{\partial \varphi}(\delta)=0$. This follows as soon as we check that for each subset $A \subset \partial S$ with diam $A \leq \delta$ the image $\partial \varphi(A)$ is a singleton. Take any two branches $b_{1}, b_{2} \in \partial \varphi(A)$ and find two branches $a_{1}, a_{2} \in A$ with $b_{i} \in \partial \varphi\left(a_{i}\right)$ for $i \in\{1,2\}$. Since $\rho_{f}\left(a_{1}, a_{2}\right) \leq \delta=f(v)$, there is a point $x \in a_{1} \cap a_{2} \cap \nu$. Then $\varphi(x) \in \lambda_{0} \cap b_{1} \cap b_{2}$ and the minimality of $\lambda_{0}$ implies that $b_{1}=b_{2}$.

Next, assume that the tower $T$ is $\downarrow$-unbounded. In this case for every $\varepsilon>0$ we can find a level $\lambda \in \operatorname{Lev}(T)$ with $g(\lambda) \leq \varepsilon$. By our hypothesis, for the level $\lambda$ there is a level $v \in \operatorname{Lev}(S)$ such that $\operatorname{lev}_{T}(\varphi(x)) \leq \lambda$ for each $x \in S$ with $\operatorname{lev}_{S}(x) \leq v$. Let $\delta=f(v)$. We claim that $\omega_{\partial \varphi}(\delta) \leq \varepsilon$. This follows as soon as we check that for each subset $A \subset \partial S$ with diam $A \leq \delta$ the image $\partial \varphi(A)$ has diameter $\leq \varepsilon$. Take any two branches $b_{1}, b_{2} \in \partial \varphi(A)$ and find two branches $a_{1}, a_{2} \in A$ with $b_{i} \in \partial \varphi\left(a_{i}\right)$ for $i \in\{1,2\}$. Since $\rho_{f}\left(a_{1}, a_{2}\right) \leq \delta$, there is a point $x \in a_{1} \cap a_{2} \cap v$. Since $\operatorname{lev}_{T}(\varphi(x)) \leq \lambda$ and $\varphi(x) \in b_{1} \cap b_{2}$, we get $\rho_{g}\left(b_{1}, b_{2}\right) \leq g(\lambda)<\varepsilon$. 
(3) Assume that for all $v \in \operatorname{Lev}(S)$ there exists $\lambda \in \operatorname{Lev}(T)$ such that $\operatorname{lev}_{S}(x) \leq v \Rightarrow \operatorname{lev}_{T}(\varphi(x)) \leq \lambda$ for all $x \in S$. The macro-uniform property of the boundary map $\partial \varphi: \partial S \Rightarrow \partial T$ follows as soon as we check that for every $\delta<\infty$ the oscillation $\omega_{\partial \varphi}(\delta)$ is finite. Find a level $v \in \operatorname{Lev}(S)$ such that $f(v) \geq \delta$. By our hypothesis, for the level $v$ there is a level $\lambda \in \operatorname{Lev}(T)$ such that $\operatorname{lev}_{T}(\varphi(x)) \leq \lambda$ for each $x \in S$ with $\operatorname{lev}_{S}(x) \leq \nu$. We claim that $\omega_{\partial \varphi}(\delta) \leq \varepsilon$ where $\varepsilon=g(\lambda)$. This follows as soon as we check that for each subset $A \subset \partial S$ with diam $A \leq \delta$ the image $\partial \varphi(A)$ has diameter $\leq \varepsilon$. Take any two branches $b_{1}, b_{2} \in \partial \varphi(A)$ and find two branches $a_{1}, a_{2} \in A$ with $b_{i} \in \partial \varphi\left(a_{i}\right)$ for $i \in\{1,2\}$. Since $\rho_{f}\left(a_{1}, a_{2}\right) \leq \delta \leq f(v)$, there is a point $x \in a_{1} \cap a_{2} \cap v$. Then $\operatorname{lev}_{T}(\varphi(x)) \leq \lambda$ and $\varphi(x) \in b_{1} \cap b_{2}$ implies that $\rho_{g}\left(b_{1}, b_{2}\right) \leq g(\lambda)=\varepsilon$.

Proposition 5.2 implies

Corollary 5.3. For a level-preserving monotone map $\varphi: S \rightarrow T$ defined on a pruned tower $S$ the induced multi-map $\partial \varphi: \partial S \Rightarrow \partial T$ is

(1) macro-uniform;

(2) bi-uniform if $\varphi_{\mathrm{Lev}}(\operatorname{Lev}(S))$ is $\downarrow$-cofinal in $\operatorname{Lev}(T)$.

Next, we establish some properties of the boundary multi-maps induced by tower immersions.

Proposition 5.4. For a tower immersion $\varphi: S \rightarrow T$ defined on a pruned tower $S$ the induced multi-map $\partial \varphi: \partial S \Rightarrow \partial T$ is

(1) a macro-uniform embedding;

(2) a bi-uniform embedding if the tower $S$ is $\downarrow$-unbounded;

(3) a macro-uniform equivalence if $\varphi(S)$ is $\downarrow$-cofinal in $T$;

(4) a bi-uniform equivalence if $S$ is $\downarrow$-unbounded and $\varphi(S)$ is $\downarrow$-cofinal in $T$.

Proof. Let $\varphi: S \rightarrow T$ be a tower immersion. It follows from the definition of $\partial \varphi$ that $(\partial \varphi)^{-1}(\partial T)=\partial S$. The boundaries $\partial S$ and $\partial T$ are endowed with the ultrametrics $\rho_{f}$ and $\rho_{g}$ induced by some scaling functions $f: \operatorname{Lev}(S) \rightarrow(0, \infty)$ and $g: \operatorname{Lev}(T) \rightarrow$ $(0, \infty)$.

(1) Corollary 5.3 implies that the boundary multi-map $\partial \varphi: \partial S \Rightarrow \partial T$ is macrouniform. It remains to check that the inverse multi-map $(\partial \varphi)^{-1}: \partial S \Rightarrow \partial T$ is macro-uniform. This is clear if the tower $S$ is $\uparrow$-bounded (in which case $\partial S$ has finite diameter). So we assume that $S$ is $\uparrow$-unbounded. The tower immersion $\varphi$, being monotone and level-preserving, induces a monotone injective map $\varphi_{\text {Lev }}: \operatorname{Lev}(S) \rightarrow$ $\operatorname{Lev}(T)$. Now we see that $\varphi_{\operatorname{Lev}}(\operatorname{Lev}(S))$ is $\uparrow$-cofinal in $\operatorname{Lev}(T)$ and the tower $T$ is $\uparrow$-unbounded.

Given any finite $\delta$ we should find a finite $\varepsilon$ such that $\omega_{(\partial \varphi)^{-1}}(\delta) \leq \varepsilon$, which means that $\operatorname{diam}(\partial \varphi)^{-1}(A) \leq \varepsilon$ for any subset $A \subset \partial T$ with diam $A \leq \delta$. Since the tower 
$T$ is $\uparrow$-unbounded, there is a level $\lambda \in \operatorname{Lev}(T)$ such that $g(\lambda) \geq \delta$. The $\uparrow$-cofinality of the set $\varphi_{\mathrm{Lev}}(\operatorname{Lev}(S))$ in $\operatorname{lev}(T)$ allows us to assume additionally that $\lambda=\varphi_{\mathrm{Lev}}(v)$ for some level $v \in \operatorname{Lev}(S)$. We claim that the finite number $\varepsilon=f(v+1)$ has the desired property. Take any two branches $b_{1}, b_{2} \in(\partial \varphi)^{-1}(A)$ and find two branches $a_{1}, a_{2} \in A$ with $b_{i} \in(\partial \varphi)^{-1}\left(a_{i}\right)$ for $i \in\{1,2\}$. The latter inclusion is equivalent to $a_{i} \in \partial \varphi\left(b_{i}\right)$. Since $\rho_{g}\left(a_{1}, a_{2}\right) \leq \operatorname{diam} A \leq \delta \leq g(\lambda)$, there is a point $y \in \lambda \cap a_{1} \cap a_{2}$.

For every $i \in\{1,2\}$ let $x_{i}$ be the unique point of the intersection $b_{i} \cap v$. It follows that $\varphi\left(x_{i}\right) \in \varphi\left(b_{i}\right) \cap \varphi(v) \subset a_{i} \cap \lambda=y$. Since $\varphi$ is a tower immersion, $\operatorname{lev}\left(x_{1} \wedge x_{2}\right) \leq \max \left\{\operatorname{lev}\left(x_{1}\right), \operatorname{lev}\left(x_{2}\right)\right\}+1=v+1$. Then $x_{1} \wedge x_{2} \subset b_{1} \cap b_{2}$ and then $\rho_{f}\left(b_{1}, b_{2}\right) \leq f\left(\operatorname{lev}\left(x_{1} \wedge x_{2}\right)\right) \leq f(v+1)=\varepsilon$.

(2) Assume that the tower $S$ is $\downarrow$-unbounded. Since the map $\varphi_{\text {Lev }}: \operatorname{Lev}(S) \rightarrow$ $\operatorname{Lev}(T)$ is injective and monotone, the set $\varphi_{\operatorname{Lev}}(\operatorname{Lev}(S))$ is $\downarrow$-cofinal in $\operatorname{Lev}(T)$ and the tower $T$ is $\downarrow$-unbounded. By Corollary 5.3, the map $\partial \varphi: \partial S \Rightarrow \partial T$ is bi-uniform and by the preceding item, the inverse multi-map $(\partial \varphi)^{-1}: \partial T \Rightarrow \partial S$ is macro-uniform. It remains to check that this map micro-uniform. Since $S$ is $\downarrow$-unbounded, for any $\varepsilon>0$ we can find a level $v \in \operatorname{Lev}(S)$ with $f(v+1) \leq \varepsilon$. Since $T$ is $\downarrow$-unbounded, we can find a level $\lambda \leq \varphi_{\text {Lev }}(\nu)$ in $T$. Repeating the argument from the preceding item we can show that the positive real number $\delta=g(\lambda)$ satisfies the inequality $\omega_{(\partial \varphi)^{-1}}(\delta) \leq \varepsilon$, witnessing that the multi-map $(\partial \varphi)^{-1}$ is micro-uniform.

(3) The third statement follows from the first one as soon as we check that $\partial \varphi(\partial S)=\partial T$ provided $\varphi(S)$ is $\downarrow$-cofinal in $T$.

If $T$ is $\downarrow$-bounded, then the ordered $\operatorname{set} \operatorname{lev}(T)$ contains the smallest element $\lambda_{0}$. Then each branch $\beta \in \operatorname{Lev}(T)$ is equal to $\uparrow y$ where $\{y\}=\beta \cap \lambda_{0}$. The cofinality of $\varphi(S)$ in $T$ implies that $\lambda_{0} \subset \varphi(S)$. Take any point $x \in S$ with $\varphi(x)=y$ and observe that $\uparrow x$ is a branch in $\partial S$ whose image $\partial \varphi(\uparrow x)=\uparrow y=\beta$.

If $T$ is $\downarrow$-unbounded, then so is the tower $S$. Let us show that the tower $T$ is pruned. Take any point $t \in T$ and use the cofinality of $\varphi(S)$ in $T$ in order to find a point $s \in S$ with $\varphi(s) \leq t$. Since $S$ is pruned, there is a point $s^{\prime} \in S$ with $s^{\prime}<s$ and the monotonicity of $\varphi$ guarantees that $\varphi\left(s^{\prime}\right)<\varphi(s) \leq t$, witnessing that $T$ is pruned.

Given any branch $\beta \in \partial T$ we are going to find a branch $\alpha \in \partial S$ with $\partial \varphi(\alpha)=\beta$. Taking into account that the tower $T$ is pruned and $\downarrow$-unbounded, we conclude that the branch $\beta$ meets all the levels of $T$. Fix a $\downarrow$-cofinal subset $L \subset \operatorname{Lev}(S)$ such that $\lambda+1 \notin L$ for every $\lambda \in L$.

For every $\lambda \in L$ pick a point $x_{\lambda} \in \lambda \cap \varphi^{-1}(\beta)$. Such a point $x_{\lambda}$ exists because $\beta$ meets the level $\varphi(\lambda)$ of $T$. Let $x_{\lambda}^{+}$be the unique point of the intersection $\uparrow x_{\lambda} \cap(\lambda+1)$.

We claim that the set $\left\{x_{\lambda}^{+}: \lambda \in L\right\}$ is linearly ordered. Indeed, take any two levels $v<\lambda$ and let $z_{\lambda}$ be the unique point of the intersection $\lambda \cap \uparrow\left(x_{v}^{+}\right)$. Taking into account that

$$
\varphi\left(z_{\lambda}\right) \geq \varphi\left(x_{v}^{+}\right) \in \uparrow \varphi\left(x_{v}^{+}\right) \subset \beta,
$$

we see that $\varphi\left(z_{\lambda}\right) \in \beta \cap \varphi(\lambda)=\left\{\varphi\left(x_{\lambda}\right)\right\}$ and hence $\varphi\left(z_{\lambda}\right)=\varphi\left(x_{\lambda}\right)$. Since $\varphi$ is a tower immersion, $\operatorname{lev}\left(z_{\lambda} \wedge x_{\lambda}\right) \leq \lambda+1$ and thus $x_{v}^{+} \leq z_{\lambda} \wedge x_{\lambda} \leq x_{\lambda}^{+}$. 
The linearly ordered subset $\left\{x_{\lambda}^{+} \mid \lambda \in L\right\}$ can be enlarged to a branch $\alpha \in \partial S$ whose image $\partial \varphi(\alpha)$ coincides with the branch $\beta$.

(4) If $\varphi(S)$ is cofinal in $T$ and the tower $S$ is $\downarrow$-unbounded, then $\partial \varphi$ is a bi-uniform equivalence, being a surjective bi-uniform embedding according to the statements (2) and (3) of Proposition 5.4.

5.3. Level subtowers. It is clear that each $\uparrow$-directed subset $S$ of a tower $T$ is a tower with respect to the partial order inherited from $T$. In this case we say that $S$ is a subtower of $T$. A typical example of a subtower of $T$ is a level subtower

$$
T^{L}=\left\{x \in T: \operatorname{lev}_{T}(x) \in L\right\},
$$

where $L \subset \operatorname{Lev}(T)$ is an $\uparrow$-cofinal subset of the level set of the tower $T$.

Proposition 5.4 implies

Corollary 5.5. Let $T$ be a pruned tower and $L$ be a $\uparrow$-cofinal subset of $\operatorname{Lev}(T)$. The multi-map did: $\partial T^{L} \Rightarrow \partial T$ induced by the identity embedding id: $T^{L} \rightarrow T$ is

(1) a macro-uniform equivalence;

(2) a bi-uniform equivalence if $L$ is $\downarrow$-cofinal in $\operatorname{Lev}(T)$.

5.4. Tower immersions induced by macro-uniform embeddings. In Proposition 5.4 we proved that for a tower immersion $\varphi: S \rightarrow T$ its boundary $\partial \varphi: \partial S \Rightarrow \partial T$ is a macro-uniform embedding. It turns out that this statement can be partly reversed.

Proposition 5.6. Let $S, T$ be pruned $\uparrow$-unbounded towers. For any macro-uniform embedding $\Phi: \partial S \Rightarrow \partial T$ there are $\downarrow$-bounded $\uparrow$-cofinal subsets $A \subset \operatorname{Lev}(S)$, $B \subset \operatorname{Lev}(T)$ and a tower immersion $\varphi: S^{A} \rightarrow T^{B}$ such that

$$
\partial \varphi=\left(\partial \operatorname{id}_{T}\right)^{-1} \circ \Phi \circ \partial \operatorname{id}_{S}
$$

where $\partial \operatorname{id}_{S}: \partial S^{A} \Rightarrow \partial S$ and $\partial \operatorname{id}_{T}: \partial T^{B} \Rightarrow \partial T$ are boundary multi-maps, induced by the identity inclusions $\operatorname{id}_{S}: S^{A} \rightarrow S$ and $\mathrm{id}_{T}: T^{B} \rightarrow T$.

Proof. Let $\Phi: \partial S \Rightarrow \partial T$ be a macro-uniform embedding. We endow the boundaries $\partial S$ and $\partial T$ of the towers $S, T$ with the ultrametrics $\rho_{f}, \rho_{g}$ induced by some scaling functions $f: \operatorname{Lev}(S) \rightarrow[0, \infty)$ and $g: \operatorname{Lev}(T) \rightarrow[0, \infty)$. Let $\alpha_{0}$ be any level of the tower $S$.

By induction we can construct two increasing sequences $A=\left\{\alpha_{n}\right\}_{n \in \omega} \subset \operatorname{Lev}(S)$ and $B=\left\{\beta_{n}\right\}_{n \in \omega} \subset \operatorname{Lev}(T)$ such that

$$
f\left(\beta_{n}\right) \geq \omega_{\Phi}\left(g\left(\alpha_{n}\right)\right) \quad \text { and } \quad g\left(\alpha_{n+1}\right) \geq \omega_{\Phi^{-1}}\left(f\left(\beta_{n}\right)\right)
$$

for all $n \geq 0$.

Now we construct a tower immersion $\varphi: S^{A} \rightarrow T^{B}$. Given any point $s \in S^{A}$, find a level $\alpha_{n}$ containing $s$ and observe that the lower cone $\downarrow s \subset S$ has diameter 
diam $\downarrow s \leq f\left(\alpha_{n}\right)$. Since diam $\Phi(\downarrow s) \leq \omega_{\Phi}\left(f\left(\alpha_{n}\right)\right) \leq g\left(\beta_{n}\right)$, we conclude that $\Phi(\downarrow s) \subset \downarrow \varphi(s)$ for a unique point $\varphi(s) \in \beta_{n}$.

It is clear that the so-defined map $\varphi: S^{A} \rightarrow T^{B}$ maps each level $\alpha_{n}, n \in \omega$, into the level $\beta_{n}$, and hence is level-preserving. The uniqueness of the point $\varphi(s)$ with $\downarrow \varphi(s) \supset \Phi(\downarrow s)$ implies that $\varphi$ is monotone.

To show that $\varphi$ is a tower immersion, take two points $s, s^{\prime} \in \alpha_{n}$ and assume that $\varphi(s)=\varphi\left(s^{\prime}\right)=t$ for some point $t \in \beta_{n} \subset T$. Then $\Phi(\downarrow s) \cup \Phi\left(\downarrow s^{\prime}\right) \subset \downarrow t$ and consequently $\downarrow s \cup \downarrow s^{\prime} \subset \Phi^{-1}(\downarrow t)$. It follows from the choice of $\alpha_{n+1}$ that

$$
\operatorname{diam}\left(\downarrow s \cup \downarrow s^{\prime}\right) \leq \operatorname{diam} \Phi^{-1}(\downarrow t) \leq f\left(\alpha_{n+1}\right),
$$

which implies that $s, s^{\prime} \in \downarrow s^{\prime \prime}$ for some point $s^{\prime \prime} \in \alpha_{n+1}$. Consequently, $\operatorname{lev}_{S_{A}}(s \wedge$ $\left.s^{\prime}\right) \leq \alpha_{n+1}$ and the level $\alpha_{n+1}$ is the successor level of $\alpha_{n}=\operatorname{lev}(s)=\operatorname{lev}\left(s^{\prime}\right)$ in the tower $S^{A}$, witnessing that the map $\varphi: S^{A} \rightarrow T^{B}$ is a tower immersion.

The definition of $\varphi$ easily implies that $\partial \varphi=\left(\operatorname{iid}_{T}\right)^{-1} \circ \Phi \circ \partial \operatorname{id}_{S}$.

By analogy we can prove

Proposition 5.7. Let $S, T$ be pruned $\uparrow$-unbounded towers. For any bi-uniform embedding $\Phi: \partial S \rightarrow \partial T$ there are $\uparrow$-cofinal subsets $A \subset \operatorname{Lev}(S), B \subset \operatorname{Lev}(T)$ and a tower immersion $\varphi: S^{A} \rightarrow T^{B}$ such that

$$
\partial \varphi=\left(\partial \operatorname{id}_{T}\right)^{-1} \circ \Phi \circ \partial \operatorname{id}_{S},
$$

where $\partial \operatorname{id}_{S}: \partial S^{A} \rightarrow \partial S$ and $\partial \operatorname{id}_{T}: \partial T^{B} \rightarrow \partial T$ are bi-uniform equivalences, induced by the identity inclusions $\operatorname{id}_{S}: S^{A} \rightarrow S$ and $\operatorname{id}_{T}: T^{B} \rightarrow T$.

5.5. Constructing tower embeddings and isomorphisms. In this subsection we describe a method of constructing tower embedding and isomorphisms.

Proposition 5.8. Let $S, T$ be pruned towers and $f: \operatorname{Lev}(S) \rightarrow \operatorname{Lev}(T)$ be a monotone (and surjective) map. If $\operatorname{Deg}_{\lambda}^{\lambda+1}(S) \leq \operatorname{deg}_{f(\lambda)}^{f(\lambda+1)}(T)\left(\right.$ and $\operatorname{deg}_{\lambda}^{\lambda+1}(S) \geq$ $\left.\operatorname{Deg}_{f(\lambda)}^{f(\lambda+1)}(T)\right)$ for each non-maximal level $\lambda \in \operatorname{Lev}(S)$, then there is a tower embedding (a tower isomorphism) $\varphi: S \rightarrow T$ such that $\varphi_{\mathrm{lev}}=f$.

Proof. A map $\varphi: A \rightarrow T$ defined on a subset $A \subset S$ will be called an $f$-map if $\operatorname{lev}_{T}(\varphi(a))=f\left(\operatorname{lev}_{S}(a)\right)$ for every $a \in A$. If, in addition, $\varphi$ is a tower embedding (isomorphism), then $\varphi$ will be called $f$-embedding ( $f$-isomorphism). The proof of Proposition 5.8 is based on the following lemma.

Lemma 5.9. For any two points $u \in S$ and $v \in T$ with $f\left(\operatorname{lev}_{S}(u)\right)=\operatorname{lev}_{T}(v)$ there is an $f$-embedding $(f$-isomorphism) $\varphi: \downarrow u \rightarrow \downarrow v$. Moreover, if for some $u_{0} \in \operatorname{pred}(u)$ and $v_{0} \in \operatorname{pred}_{f\left(\operatorname{lev} u_{0}\right)}(v)$ we are given with a tower $f$-embedding $(f$ isomorphism) $\varphi_{0}: \downarrow u_{0} \rightarrow \downarrow v_{0}$, then the map $\varphi$ can be chosen so that $\varphi \mid \downarrow u_{0}=\varphi_{0}$. 
Proof. For every level $\lambda \leq \operatorname{lev}_{S}(u)$ of $S$ consider the subtower $S_{\lambda}(u)=\{s \in \downarrow u \mid$ $\operatorname{lev}(s) \geq \lambda\}$ having finitely many levels. By induction we are going to construct an $f$-embedding $\varphi_{\lambda}: S_{\lambda}(u) \rightarrow T$ so that $\varphi_{\lambda-1}$ extends $\varphi_{\lambda}$.

If $\lambda=\operatorname{lev}_{S}(u)$, then $S_{\lambda}(u)=\{u\}$ and we can put $\varphi_{\lambda}(u)=v$. Assume that for some level $\lambda<\operatorname{lev}_{S}(u)$ of $S$ an $f$-embedding $\varphi_{\lambda+1}: S_{\lambda+1}(u) \rightarrow T$ has been constructed. Observe that

$$
S_{\lambda}(u)=S_{\lambda+1}(u) \cup \bigcup\{\operatorname{pred}(x) \mid x \in(\lambda+1) \cap \downarrow u\} .
$$

By our assumption, for every $x \in(\lambda+1) \cap \downarrow u$, we get

$$
\operatorname{deg}(x) \leq \operatorname{Deg}_{\lambda}^{\lambda+1}(S) \leq \operatorname{deg}_{f(\lambda)}^{f(\lambda+1)}(T) \leq \operatorname{deg}_{f(\lambda)}^{f(\lambda+1)}(f(x)) .
$$

Consequently, we can find an injective map $\psi_{x}: \operatorname{pred}_{\lambda}(x) \rightarrow \operatorname{pred}_{f(\lambda)}(f(x))$. Moreover, if $\operatorname{deg}_{\lambda}(x)=\operatorname{deg}_{f(\lambda)}(f(x))$, then we can take the map $\psi_{x}$ to be bijective. If for some $u_{0} \in \operatorname{pred}(u)$ and $v_{0} \in \operatorname{pred}_{f\left(\operatorname{lev} u_{0}\right)}(v)$ we are given with a tower $f$-embedding ( $f$-isomorphism) $\varphi_{0}: \downarrow u_{0} \rightarrow \downarrow v_{0}$, then we can assume that $\psi_{x}=\varphi_{0} \mid \operatorname{pred}(x)$ if $x \leq u_{0}$.

Now define the $f$-embedding $\varphi_{\lambda}: S_{\lambda} \rightarrow T$ by letting $\varphi_{\lambda} \mid S_{\lambda+1}=\varphi_{\lambda+1}$ and $\varphi_{\lambda} \mid \operatorname{pred}_{\lambda}(x)=\psi_{x}$ for $x \in(\lambda+1) \cap \downarrow u$. This completes the inductive step.

One can readily check that the $f$-embedding $\varphi: \downarrow u \rightarrow \downarrow v$ defined by $\varphi \mid S_{\lambda}(u)=$ $\varphi_{\lambda}$ for levels $\lambda \leq \operatorname{lev}_{S}(u)$ of $S$ has the required properties.

Now let us return to the proof of Proposition 5.8. Fix any point $\theta_{S} \in S$ and for every level $\lambda \geq \operatorname{lev}_{S}\left(\theta_{S}\right)$ of the tower $S$ denote by $u_{\lambda}$ the unique point of the intersection $\uparrow \theta_{S} \cap \lambda$. Choose any point $\theta_{T}$ at the level $f\left(\operatorname{lev}_{S}\left(\theta_{S}\right)\right) \subset T$ and for every level $\lambda \geq \operatorname{lev}_{T}\left(\theta_{T}\right)$ denote by $v_{\lambda}$ the unique point of the intersection $\lambda \cap \uparrow \theta_{T}$.

For the initial level $\lambda=\operatorname{lev}_{S}\left(\theta_{S}\right)$ we can apply the first part of Lemma 5.9 in order to find an $f$-embedding (an $f$-isomorphism) $\varphi_{\lambda}: \downarrow u_{\lambda} \rightarrow \downarrow v_{f(\lambda)}$. Applying inductively the second part of Lemma 5.9, for every level $\lambda>\operatorname{lev}_{S}\left(\theta_{S}\right)$ of $S$ we can find an $f$-embedding ( $f$-isomorphism) $\varphi_{\lambda}: \downarrow u_{\lambda} \rightarrow \downarrow v_{f(\lambda)}$ such that $\varphi_{\lambda} \mid \downarrow u_{\lambda-1}=$ $\varphi_{\lambda-1}$.

After completing the inductive construction, we define an $f$-embedding ( $f$-isomorphism) $\varphi: S \rightarrow T$ by letting $\varphi \mid \downarrow u_{\lambda}=\varphi_{\lambda}$ for $\lambda \geq \operatorname{lev}_{S}\left(\theta_{S}\right)$. The $f$-embedding $\varphi$ is well defined because $S$ is upward directed and hence $S=$ $\bigcup_{\lambda \geq \operatorname{lev}_{S}\left(\theta_{S}\right)} \downarrow u_{\lambda}$.

Applying Proposition 5.8 to homogeneous towers we get

Corollary 5.10. Two homogeneous towers $S, T$ are isomorphic if and only if there is an order isomorphism $f: \operatorname{Lev}(S) \rightarrow \operatorname{Lev}(T)$ such that $\operatorname{deg}_{\lambda}^{\lambda+1}(S)=\operatorname{deg}_{f(\lambda)}^{f(\lambda+1)}(T)$ for each non-maximal level $\lambda \in \operatorname{Lev}(S)$. 


\section{The key lemma}

The principal result of this section is Lemma 6.1, which is the most difficult result of this paper. This lemma allows us to construct immersions between $\downarrow$-bounded towers and will be used in the proof of Theorems 5 and 6 in Sections 7 and 8.

It follows from Corollary 5.5 that the boundary $\partial T$ of each tower $T$ is macrouniformly equivalent to the boundary $\partial T^{L}$ of the level subtower $T^{L}$ for any $\uparrow$-cofinal subset $L \subset \operatorname{Lev}(T)$. The subset $L$ can be chosen to be $\downarrow$-bounded in $\operatorname{Lev}(T)$, which implies that the level subtower $T^{L}$ is $\downarrow$-bounded. Therefore, for studying the macrouniform structure of ultrametric spaces it suffices to restrict ourselves by $\downarrow$-bounded $\uparrow$-unbounded towers $T$.

In this case the level set $\operatorname{Lev}(T)$ of $T$ has the smallest element and can be canonically labeled by finite ordinals. For $k \in \omega$ by $\operatorname{Lev}_{k}(T)$ we denote the $k$-th level of $T$. The identification of $\operatorname{Lev}(T)$ with $\omega$ defines the canonical scaling function id: $\operatorname{Lev}(T) \rightarrow \omega \subset[0, \infty)$ that induces the canonical ultrametric $\rho_{\text {id }}$ on the boundary $\partial T$ of $T$. Observe that $\partial T$ can be identified with the smallest $\operatorname{level}^{\operatorname{Lev}_{0}(T)}$ of $T$.

Lemma 6.1. For a $\downarrow$-bounded tower $T$ and $a \downarrow$-bounded homogeneous tower $H$ there is a surjective tower immersion $\varphi: T \rightarrow H$ if the two inequalities

(1) $\operatorname{deg}_{0}^{k}(T) \geq 4^{k+5} \cdot \operatorname{deg}_{0}^{k-1}(H)$,

(2) $\operatorname{deg}_{0}^{k}(H) \geq 4^{k} \cdot \operatorname{Deg}_{0}^{k}(T)$

hold for every $k \in \mathbb{N}$.

Proof. First we introduce some notation.

A subset $A$ of the tower $T$ will be called a trapezium if $A=\downarrow P$ for some nonempty subset $P \subset \operatorname{pred}(v)$ of parents of some point $v \in T$, called the vertex of the trapezium $A$ and denoted by vx $(A)$. It is easy to see that $\{\operatorname{vx}(A)\} \cup \downarrow P$ is a subtower of $T$. The set $P$ generating the trapezium $A=\downarrow P$ is called the plateau of the trapezium. For the plateau $P$ let $\operatorname{deg}_{0}(P)=\left|\downarrow P \cap \operatorname{Lev}_{0}(T)\right|$ be the cardinality of the "base" $\downarrow P \cap \operatorname{Lev}_{0}(T)$ of the trapezium $\downarrow P$.

A map $\varphi: \downarrow P \rightarrow H$ from a trapezium $\downarrow P \subset S$ to the tower $H$ will be called an admissible immersion if

- $\varphi=\phi \mid \downarrow P$ for some tower immersion $\phi:\{\operatorname{vx}(\downarrow P)\} \cup \downarrow P \rightarrow H$,

- $\varphi(P)=\{t\}$ for some $t \in T$,

- $\varphi(\downarrow P)=\downarrow t$.

Let $\varepsilon_{k}=\frac{1}{4^{k}}, k \in \mathbb{N}$, and observe that

$$
\prod_{k=1}^{\infty} \frac{1+\varepsilon_{k}}{1-\varepsilon_{k}}<2 .
$$

Lemma 6.1 will be derived from the following 
Claim 6.2. For any $k \in \mathbb{N}$, a trapezium $\downarrow A_{k} \subset T$, and a vertex $w \in H$ at the height $k=\operatorname{lev}\left(A_{k}\right)=\operatorname{lev}(w)$ there is an admissible immersion $\varphi: \downarrow A_{k} \rightarrow \downarrow w$ provided that

$$
4 \leq 8 \cdot \prod_{i=k+1}^{\infty} \frac{1-\varepsilon_{i}}{1+\varepsilon_{i}} \leq \frac{\operatorname{deg}_{0}\left(A_{k}\right)}{\operatorname{deg}_{0}^{k}(H)} \leq 16 \prod_{i=k+1}^{\infty} \frac{1+\varepsilon_{i}}{1-\varepsilon_{i}} \leq 32 .
$$

Proof. The proof is by induction on $k$. If $k=0$, then $\downarrow A_{k}=A_{k}$ and the constant map $\varphi: A_{k} \rightarrow\{w\} \subset H$ is the required immersion.

Assume that the claim has been proved for some $k-1 \in \omega$. Fix a trapezium $\downarrow A_{k} \subset S$ and a point $w \in T$ with $\operatorname{lev}_{S}\left(A_{k}\right)=\operatorname{lev}_{T}(w)=k$ so that the upper and lower bounds from Claim 6.2 hold.

Since $\operatorname{deg}_{0}\left(A_{k}\right)=\sum_{a \in A_{k}} \operatorname{deg}_{0}(a)$, for every point $a \in A_{k}$ we can choose an integer number $d_{a}$ such that

$$
\left|d_{a}-\operatorname{deg}_{k-1}^{k}(H) \frac{\operatorname{deg}_{0}(a)}{\operatorname{deg}_{0}\left(A_{k}\right)}\right| \leq 1
$$

and $\sum_{a \in A_{k}} d_{a}=\operatorname{deg}_{k-1}^{k}(H)=\operatorname{deg}(w)$.

Claim 6.3. For every $a \in A_{k}$,

$$
\frac{\operatorname{deg}_{k-1}^{k}(H)}{\operatorname{deg}_{0}\left(A_{k}\right)}\left(1-\varepsilon_{k}\right) \leq \frac{d_{a}}{\operatorname{deg}_{0}(a)} \leq \frac{\operatorname{deg}_{k-1}^{k}(H)}{\operatorname{deg}_{0}\left(A_{k}\right)}\left(1+\varepsilon_{k}\right) .
$$

Proof. It follows from the choice of $d_{a}$ that

$$
\frac{d_{a}}{\operatorname{deg}_{0}(a)} \leq \frac{\operatorname{deg}_{k-1}^{k}(H)}{\operatorname{deg}_{0}\left(A_{k}\right)}+\frac{1}{\operatorname{deg}_{0}(a)}=\frac{\operatorname{deg}_{k-1}^{k}(H)}{\operatorname{deg}_{0}\left(A_{k}\right)} \cdot\left(1+\frac{\operatorname{deg}_{0}\left(A_{k}\right)}{\operatorname{deg}_{k-1}^{k}(H) \cdot \operatorname{deg}_{0}(a)}\right) .
$$

The upper bound in Claim 6.2 implies

$$
\frac{\operatorname{deg}_{0}\left(A_{k}\right)}{\operatorname{deg}_{k-1}^{k}(H) \cdot \operatorname{deg}_{0}(a)} \leq \frac{32 \cdot \operatorname{deg}_{0}^{k}(H)}{\operatorname{deg}_{k-1}^{k}(H) \cdot \operatorname{deg}_{0}(a)} \leq \frac{32 \cdot \operatorname{deg}_{0}^{k-1}(H)}{\operatorname{deg}_{0}^{k}(T)} \leq \frac{1}{4^{k}}=\varepsilon_{k} .
$$

The last inequality follows from the condition (1) of Lemma 6.1.

This proves the upper bound of Claim 6.3. By analogy we can prove the lower bound.

Claim 6.3, the upper bound of Claim 6.2 and the condition (1) of Lemma 6.1 imply

$$
\begin{aligned}
d_{a} \geq \operatorname{deg}_{0}(a) \frac{\operatorname{deg}_{k-1}^{k}(H)}{\operatorname{deg}_{0}\left(A_{k}\right)}\left(1-\varepsilon_{k}\right) & \geq \frac{\operatorname{deg}_{0}^{k}(T) \cdot \operatorname{deg}_{k-1}^{k}(H)}{32 \operatorname{deg}_{0}^{k}(H)} \frac{1}{2} \\
& \geq \frac{4^{k+5} \cdot \operatorname{deg}_{0}^{k-1}(H)}{64 \cdot \operatorname{deg}_{0}^{k-1}(H)} \geq 4^{k-1}>0 .
\end{aligned}
$$


For every $a \in A_{k}$ write the set $\operatorname{pred}(a)$ of parents of $a$ in the tower $T$ as the disjoint union $\operatorname{pred}(a)=\bigcup \mathcal{A}_{a}$ of a family $\mathcal{A}_{a}$ containing $d_{a}$ sets such that for every $A_{k-1} \in \mathcal{A}_{a}$ we get

$$
\left|\operatorname{deg}_{0}\left(A_{k-1}\right)-\frac{\operatorname{deg}_{0}(a)}{d_{a}}\right| \leq \operatorname{Deg}_{0}^{k-1}(T) .
$$

Claim 6.4. For each set $A_{k-1} \in \mathcal{A}_{a}$ the upper and lower bounds of Claim 6.2 are satisfied for $k-1$.

Proof. If $k=1$, then

$$
\left|\operatorname{deg}_{0}\left(A_{0}\right)-\frac{\operatorname{deg}_{0}(a)}{d_{a}}\right| \leq \operatorname{Deg}_{0}^{0}(T)=1
$$

and by Claim 6.3 and the inductive assumption:

$$
\begin{aligned}
\operatorname{deg}_{0}\left(A_{0}\right) & \leq \frac{\operatorname{deg}_{0}(a)}{d_{a}}+1 \\
& \leq \frac{\operatorname{deg}_{0}\left(A_{1}\right)}{\operatorname{deg}_{0}^{1}(H)\left(1-\varepsilon_{1}\right)}+1 \\
& \leq \frac{\operatorname{deg}_{0}\left(A_{1}\right)}{\operatorname{deg}_{0}^{1}(H)\left(1-\varepsilon_{k}\right)}\left(1+\frac{\operatorname{deg}_{0}^{1}(H)}{\operatorname{deg}_{0}\left(A_{1}\right)}\right) \\
& \leq \frac{\operatorname{deg}_{0}\left(A_{1}\right)}{\operatorname{deg}_{0}^{1}(H)\left(1-\varepsilon_{1}\right)}\left(1+\frac{1}{4}\right) \\
& \leq 16 \prod_{i=1}^{\infty} \frac{1+\varepsilon_{i}}{1-\varepsilon_{i}} .
\end{aligned}
$$

By analogy, we can prove the lower bound

$$
\operatorname{deg}_{0}\left(A_{0}\right) \geq \frac{\operatorname{deg}_{0}\left(A_{1}\right)}{\operatorname{deg}_{0}^{1}(H)} \cdot \frac{1-\varepsilon_{1}}{1+\varepsilon_{1}} \geq 8 \prod_{i=1}^{\infty} \frac{1-\varepsilon_{i}}{1+\varepsilon_{i}} .
$$

Next, assume that $k>1$. Then by Claim 6.3:

$$
\begin{aligned}
\frac{\operatorname{deg}_{0}\left(A_{k-1}\right)}{\operatorname{deg}_{0}^{k-1}(H)} & \leq \frac{1}{\operatorname{deg}_{0}^{k-1}(H)} \cdot \frac{\operatorname{deg}_{0}(a)}{d_{a}}+\frac{\operatorname{Deg}_{0}^{k-1}(T)}{\operatorname{deg}_{0}^{k-1}(H)} \\
& \leq \frac{1}{\operatorname{deg}_{0}^{k-1}(H)} \cdot \frac{\operatorname{deg}_{0}\left(A_{k}\right)}{\operatorname{deg}_{k-1}^{k}(H)\left(1-\varepsilon_{k}\right)}+\frac{\operatorname{Deg}_{0}^{k-1}(T)}{\operatorname{deg}_{0}^{k-1}(H)} \\
& \leq \frac{\operatorname{deg}_{0}\left(A_{k}\right)}{\operatorname{deg}_{0}^{k}(H)\left(1-\varepsilon_{k}\right)}\left(1+\frac{\operatorname{Deg}_{0}^{k-1}(T) \operatorname{deg}_{0}^{k}(H)}{\operatorname{deg}_{0}^{k-1}(H) \operatorname{deg}_{0}\left(A_{k}\right)}\right)
\end{aligned}
$$


and

$$
\begin{aligned}
\frac{\operatorname{Deg}_{0}^{k-1}(T) \operatorname{deg}_{0}^{k}(H)}{\operatorname{deg}_{0}^{k-1}(H) \operatorname{deg}_{0}\left(A_{k}\right)} & =\frac{\operatorname{Deg}_{0}^{k-1}(T) \operatorname{deg}_{k-1}^{k}(H)}{\operatorname{deg}_{0}\left(A_{k}\right)} \\
& \leq \frac{\operatorname{Deg}_{0}^{k-1}(T) \operatorname{deg}_{k-1}^{k}(H)}{4 \operatorname{deg}_{0}^{k}(H)}=\frac{\operatorname{Deg}_{0}^{k-1}(T)}{4 \operatorname{deg}_{0}^{k-1}(H)} \leq \frac{1}{4 \cdot 4^{k}} \leq \varepsilon_{k}
\end{aligned}
$$

by the lower bound from Claim 6.2 and the condition (2) of Lemma 6.1. Then

$$
\begin{aligned}
\frac{\operatorname{deg}_{0}\left(A_{k-1}\right)}{\operatorname{deg}_{0}^{k-1}(H)} & \leq \frac{\operatorname{deg}_{0}\left(A_{k}\right)}{\operatorname{deg}_{0}^{k}(H)} \cdot \frac{1+\varepsilon_{k}}{1-\varepsilon_{k}} \\
& \leq 16 \cdot\left(\prod_{i=k+1}^{\infty} \frac{1+\varepsilon_{i}}{1-\varepsilon_{i}}\right) \cdot \frac{1+\varepsilon_{k}}{1-\varepsilon_{k}}=16 \cdot \prod_{i=k}^{\infty} \frac{1+\varepsilon_{i}}{1-\varepsilon_{i}}
\end{aligned}
$$

By analogy, we can prove that

$$
\frac{\operatorname{deg}_{0}\left(A_{k-1}\right)}{\operatorname{deg}_{0}^{k-1}(H)} \geq \frac{\operatorname{deg}_{0}\left(A_{k}\right)}{\operatorname{deg}_{0}^{k}(H)} \cdot \frac{1-\varepsilon_{k}}{1+\varepsilon_{k}} \geq 8 \cdot \prod_{i=k}^{\infty} \frac{1+\varepsilon_{i}}{1-\varepsilon_{i}}
$$

The family $\mathcal{A}=\bigcup_{a \in A_{k}} \mathcal{A}_{a}$ has cardinality $|\mathcal{A}|=\sum_{a \in A_{k}}\left|\mathcal{A}_{a}\right|=\sum_{a \in A_{k}} d_{a}=$ $\operatorname{deg}(w)$ and hence we can find a bijective map $f: \mathcal{A} \rightarrow \operatorname{pred}(w)$. By the inductive assumption and Claim 6.4, for each set $A^{\prime} \in \mathcal{A}$ we can find an admissible immersion $\varphi_{A^{\prime}}: \downarrow A^{\prime} \rightarrow \downarrow f\left(A^{\prime}\right)$. Now define the admissible immersion $\varphi: \downarrow P \rightarrow \downarrow w$ by letting

$$
\varphi(x)= \begin{cases}\varphi_{A^{\prime}}(x) & \text { if } x \in \downarrow A^{\prime} \text { for some } A^{\prime} \in \mathcal{A}, \\ w & \text { if } x \in A_{k} .\end{cases}
$$

This completes the proof of Claim 6.2.

Now we are able to complete the proof of Lemma 6.1. Let $\left(a_{k}\right)_{k \in \omega}$ and $\left(b_{k}\right)_{k \in \omega}$ be two branches of the towers $T$ and $H$, respectively. For every $k \in \omega$ choose a subset $A_{k} \subset \operatorname{pred}\left(a_{k+1}\right)$ such that $a_{k} \in A_{k}$ and

$$
11 \leq \frac{\operatorname{deg}_{0}\left(A_{k}\right)}{\operatorname{deg}_{0}^{k}(H)} \leq 13
$$

Such a choice of $A_{k}$ is always possible because $\operatorname{deg}_{0}\left(a_{k+1}\right) \geq \operatorname{deg}_{0}^{k+1}(T) \geq$ $4^{k+6} \operatorname{deg}_{0}^{k}(H)$ by the condition (1) of Lemma 6.1 and $\frac{\operatorname{Deg}_{0}^{k}(T)}{\operatorname{deg}_{0}^{k}(H)} \leq \frac{1}{4^{k}} \leq 1$ by the condition (2) of Lemma 6.1.

By induction on $k \in \omega$ we shall construct a tower immersion $\varphi_{k}: \downarrow A_{k} \rightarrow \downarrow b_{k}$ such that $\varphi_{k-1}=\varphi_{k} \mid \downarrow A_{k-1}$. 
For $k=0$ the constant map $\varphi_{0}: A_{0} \rightarrow\left\{b_{0}\right\}$ is the desired immersion. Assume that for some $k \in \omega$ an immersion $\varphi_{k}: \downarrow A_{k} \rightarrow \downarrow b_{k}$ has been constructed. Consider the trapezium $\downarrow A$ with the plateau

$$
A=\left(\operatorname{Lev}_{k}(T) \cap \downarrow A_{k+1}\right) \backslash A_{k}
$$

in the tower $T$. Also consider the trapezium $\downarrow B$ with plateau $B=\operatorname{pred}\left(b_{k+1}\right) \backslash\left\{b_{k}\right\}$ in the homogeneous tower $H$. It is clear that $\operatorname{deg}_{0}(A)=\operatorname{deg}_{0}\left(A_{k+1}\right)-\operatorname{deg}_{0}\left(A_{k}\right)$ and $|B|=\operatorname{deg}_{k}^{k+1}(H)-1$. Observe that

$$
\begin{aligned}
\operatorname{deg}_{k}^{k+1}(H)=\frac{\operatorname{deg}_{0}^{k+1}(H)}{\operatorname{deg}_{0}^{k}(H)} & \geq 4^{k+1} \frac{\operatorname{Deg}^{k+1}(T)}{\operatorname{deg}_{0}^{k}(H)} \\
& \geq 4^{k+1} \frac{4^{k+6} \operatorname{deg}_{0}^{k}(H)}{\operatorname{deg}_{0}^{k}(H)}=4^{2 k+7} \geq 4^{7} .
\end{aligned}
$$

Write $A$ as the disjoint union $A=\bigcup_{b \in B} A_{b}$ of subsets $A_{b} \subset A$ such that

$$
\left|\operatorname{deg}_{0}\left(A_{b}\right)-\frac{\operatorname{deg}_{0}(A)}{|B|}\right| \leq \operatorname{Deg}_{0}^{k}(T)
$$

for every $b \in B$. It follows from the condition (2) of Lemma 6.1 that

$$
\begin{aligned}
\frac{\operatorname{deg}_{0}\left(A_{b}\right)}{\operatorname{deg}_{0}^{k}(H)} & \leq \frac{1}{\operatorname{deg}_{0}^{k}(H)}\left(\frac{\operatorname{deg}_{0}(A)}{\operatorname{deg}_{k}^{k+1}(H)-1}+\operatorname{Deg}_{0}^{k}(T)\right) \\
& \leq \frac{1}{\operatorname{deg}_{0}^{k}(H)} \cdot \frac{\operatorname{deg}_{k}^{k+1}(H)}{\operatorname{deg}_{k}^{k+1}(H)-1} \cdot \frac{\operatorname{deg}_{0}\left(A_{k+1}\right)}{\operatorname{deg}_{k}^{k+1}(H)}+\frac{\operatorname{Deg}_{0}^{k}(T)}{\operatorname{deg}_{0}^{k}(H)} \\
& \leq \frac{4^{7}}{4^{7}-1} \cdot \frac{\operatorname{deg}_{0}\left(A_{k+1}\right)}{\operatorname{deg}_{0}^{k+1}(H)}+\frac{1}{4^{k}} \leq \frac{14}{13} \cdot 13+1<16 .
\end{aligned}
$$

On the other hand,

$$
\begin{aligned}
\frac{\operatorname{deg}_{0}\left(A_{b}\right)}{\operatorname{deg}_{0}^{k}(H)} & \geq \frac{1}{\operatorname{deg}_{0}^{k}(H)}\left(\frac{\operatorname{deg}_{0}(A)}{\operatorname{deg}_{k}^{k+1}(H)-1}-\operatorname{Deg}_{0}^{k}(T)\right) \\
& \geq \frac{1}{\operatorname{deg}_{0}^{k}(H)} \cdot \frac{\operatorname{deg}_{0}\left(A_{k+1}\right)-\operatorname{deg}_{0}\left(A_{k}\right)}{\operatorname{deg}_{k}^{k+1}(H)}-\frac{\operatorname{Deg}_{0}^{k}(T)}{\operatorname{deg}_{0}^{k}(H)} \\
& \geq \frac{11 \operatorname{deg}_{0}^{k+1}(H)-13 \operatorname{deg}_{0}^{k}(H)}{\operatorname{deg}_{0}^{k+1}(H)}-\frac{1}{4^{k}} \geq 11-\frac{13}{4^{7}}-1 \geq 8 .
\end{aligned}
$$

The above two inequalities imply that the trapezium $\downarrow A_{b}$ satisfies the upper and lower bounds of Claim 6.2, which yields an admissible immersion $\varphi_{b}: \downarrow A_{b} \rightarrow \downarrow b$. 
The immersions $\varphi_{b}$ compose the immersion $\varphi_{k+1}: \downarrow A_{k+1} \rightarrow \downarrow b_{k+1}$ defined by the formula

$$
\varphi_{k+1}(x)= \begin{cases}\varphi_{k}(x) & \text { if } x \in \downarrow A_{k}, \\ \varphi_{b}(x) & \text { if } x \in \downarrow A_{b} \text { for some } b \in B .\end{cases}
$$

Since $\varphi_{k}=\varphi_{k+1} \mid \downarrow A_{k}$ for all $k \in \omega$ we can define an immersion $\varphi: T \rightarrow H$ letting $\varphi \mid \downarrow a_{k}=\varphi_{k}$ for $k \in \omega$.

\section{Proof of Theorem 5 (macro-uniform characterization of the Cantor bi-cube)}

The "only if" part of Theorem 5 follows from Lemmas 3.1 and 3.2. To prove the "if" part, assume that a metric space $X$ has macro-uniform dimension zero and for some $\delta>0$ we get $\Theta_{\delta}^{\varepsilon}(X)<\infty$ for all $\varepsilon \geq \delta$ and $\lim _{\varepsilon \rightarrow \infty} \theta_{\delta}^{\varepsilon}(X)=\infty$.

Let $\lambda_{0}=\delta$ and $m_{0}=0$. By induction we can construct increasing sequences $\left(\lambda_{k}\right)_{k=0}^{\infty} \subset(0,+\infty)$ and $\left(m_{k}\right)_{k=0}^{\infty} \subset \omega$ such that $\theta_{\delta}^{\lambda_{k}}(X) \geq 4^{k+5} \cdot 2^{m_{k-1}}$ and $2^{m_{k}} \geq 4^{k} \cdot \Theta_{\delta}^{\lambda_{k}}(X)$ for all $k \in \mathbb{N}$.

Now define $L=\left\{\lambda_{n}\right\}_{n \in \mathbb{N}} \subset(0, \infty)$ and consider the canonical $L$-tower $T_{X}^{L}=$ $\left\{\left(C_{\lambda}(x), \lambda\right) \mid x \in X, \lambda \in L\right\}$ of the metric space $X$. Its level set $\operatorname{Lev}\left(T_{X}^{L}\right)$ can be identified with the set $L$. By Corollary 4.7, the canonical map

$$
C_{L}: X \rightarrow \partial T_{X}^{L}, \quad C_{L}: x \mapsto C_{L}(x)=\left\{\left(C_{\lambda}(x), \lambda\right) \mid \lambda \in L\right\},
$$

is a macro-uniform equivalence.

Next, consider an $\downarrow$-unbounded binary tower $T_{2}$. Its level-set $\operatorname{Lev}\left(T_{2}\right)$ can be identified with $\mathbb{Z}$ and we can consider the level subtower $T_{2}^{M} \subset T_{2}$ where $M=$ $\left\{m_{k}\right\}_{k \in \omega} \subset \mathbb{Z}$. By Corollary 5.5, the boundary multi-map $\partial \operatorname{id}_{T_{2}^{M}}: \partial T_{2}^{M} \Rightarrow \partial T_{2}=$ $2^{<\mathbb{Z}}$ induced by the identity embedding $\operatorname{id}_{T_{2}^{M}}: T_{2}^{M} \rightarrow T_{2}$ is a macro-uniform equivalence.

Observe that $H=T_{2}^{M}$ is a homogeneous tower and

$$
\operatorname{deg}_{0}^{k}\left(T_{2}^{M}\right)=2^{m_{k}}, \quad \operatorname{deg}_{0}^{k}\left(T_{X}^{L}\right)=\theta_{\delta}^{\lambda_{k}}(X), \quad \operatorname{Deg}_{0}^{k}\left(T_{X}^{L}\right)=\Theta_{\delta}^{\lambda_{k}}(X),
$$

which allows us to apply Lemma 6.1 to constructing a surjective tower immersion $\varphi: T_{X}^{L} \rightarrow T_{2}^{M}$. By Proposition 5.4(3), $\varphi$ induces a macro-uniform equivalence $\partial \varphi: \partial T_{X}^{L} \Rightarrow \partial T_{2}^{M}$. Finally we obtain a macro-uniform equivalence between $X$ and the Cantor bi-cube $2^{<\mathbb{Z}}$ as the composition of the macro-uniform equivalences

$$
X \sim \partial T_{X}^{L} \sim \partial T_{2}^{M} \sim \partial T_{2}=2^{<\mathbb{Z}} .
$$




\section{Proof of Theorem 6 (bi-uniform characterization of the Cantor bi-cube)}

The "only if" part of Theorem 6 easily follows from Lemmas 3.1 and 3.2. To prove the "if" part, assume that $X$ is a complete metric space of bi-uniform dimension zero such that for every $0<\delta \leq \varepsilon<\infty$ the number $\Theta_{\delta}^{\varepsilon}(X)$ is finite and for every $0<\varepsilon<\infty$

$$
\lim _{\delta \rightarrow+0} \theta_{\delta}^{\varepsilon}=\infty=\lim _{\delta \rightarrow+\infty} \theta_{\omega}^{\delta}(X) .
$$

Let $\lambda_{0}=1$ and $m_{0}=0$. By induction construct increasing sequences $\left(\lambda_{k}\right)_{k=0}^{\infty} \subset$ $[1, \infty)$ and $\left(m_{k}\right)_{k=0}^{\infty} \subset \omega$ such that for every $k \in \omega$ the following conditions hold:

(i) $\theta_{\lambda_{0}}^{\lambda_{k}}(X) \geq 4^{k+5} \cdot 2^{m_{k-1}}$,

(ii) $2^{m_{k}} \geq 4^{k} \Theta_{\lambda_{0}}^{\lambda_{k}}(X)$.

By reverse induction, construct sequences $\left(\lambda_{k}\right)_{k=-\infty}^{1} \subset(0,1)$ and $\left(m_{k}\right)_{k=-\infty}^{1} \subset$ $\mathbb{Z}$ such that

(iii) $\lambda_{k-1}<\lambda_{k}$ and $m_{k-1}<m_{k}$ for each $k \leq 0$,

(iv) $\lim _{k \rightarrow-\infty} \lambda_{k}=0, \lim _{k \rightarrow-\infty} m_{k}=-\infty$,

(v) $\Theta_{\lambda_{k}}^{\lambda_{k+1}}(X) \leq 2^{m_{k}-m_{k-1}} \leq \theta_{\lambda_{k-1}}^{\lambda_{k}}(X)$.

For the subset $L=\left\{\lambda_{n} \mid n \in \mathbb{Z}\right\} \subset(0,+\infty)$, consider the canonical $L$-tower $T_{X}^{L}=\left\{\left(C_{\lambda}(x), \lambda\right) \mid x \in X, \lambda \in L\right\}$ of the metric space $X$. By Corollary 4.7(3), the canonical map

$$
C_{L}: X \rightarrow \partial T_{X}^{L}, \quad C_{L}: x \mapsto C_{L}(x)=\left\{\left(C_{\lambda}(x), \lambda\right) \mid \lambda \in L\right\},
$$

is a bi-uniform equivalence.

Next, consider an $\downarrow$-unbounded binary tower $T_{2}$. Its level-set $\operatorname{Lev}\left(T_{2}\right)$ can be identified with $\mathbb{Z}$ and we can consider its level subtower $T_{2}^{M} \subset T_{2}$ where $M=$ $\left\{m_{k}\right\}_{k \in \mathbb{Z}} \subset \mathbb{Z}$. By Corollary 5.5, the boundary map $\partial_{i_{2}} T_{2}^{M}: \partial T_{2}^{M} \Rightarrow \partial T_{2}=2^{<\mathbb{Z}}$ induced by the identity embedding $\operatorname{id}_{T_{2}^{M}}: T_{2}^{M} \rightarrow T_{2}$ is a bi-uniform equivalence.

For every $n \in \mathbb{Z}$ let $L_{n}=\left\{\lambda_{k} \mid k \geq n\right\}$ and $M_{n}=\left\{m_{k} \mid k \geq n\right\}$. Repeating the argument of the proof of Theorem 5 and applying Lemma 6.1, we can find a surjective tower immersion $\varphi_{0}: T_{X}^{L_{0}} \rightarrow T_{2}^{M_{0}}$. Now our aim is to extend the immersion $\varphi_{0}$ to a tower immersion $\varphi: T_{X}^{L} \rightarrow T_{2}^{M}$.

By induction we define surjective tower immersions $\varphi_{k}: T_{X}^{L_{k}} \rightarrow T_{2}^{M_{k}}, k \leq 0$, such that $\varphi_{k-1} \mid T_{X}^{L_{k}}=\varphi_{k}$ for all $k \leq 0$.

Assuming that for some $k \leq 0$ a surjective tower immersion $\varphi_{k}: T_{X}^{L_{k}} \rightarrow T_{2}^{M_{k}}$ has been defined, we construct a tower immersion $\varphi_{k-1}: T_{X}^{L_{k-1}} \rightarrow T_{2}^{M_{k-1}}$ as follows. Since $\varphi_{k}$ is a tower immersion, for every point $y \in T_{2}^{M_{k}}$ at the lowest level $m_{k}$ of the tower $T_{2}^{M_{k}}$ the preimage $\varphi_{k}^{-1}(y)$ lies in the set $\operatorname{pred}_{\lambda_{k}}(s)=\lambda_{k} \cap \downarrow s$ of parents 
of some point $s \in \lambda_{k+1}$. Consequently, $\left|\varphi_{k}^{-1}(y)\right| \leq \operatorname{deg}_{\lambda_{k}}(s) \leq \operatorname{Deg}_{\lambda_{k}}^{\lambda_{k+1}}\left(T_{X}\right)=$ $\Theta_{\lambda_{k}}^{\lambda_{k+1}}(X)$. By the choice of $\lambda_{k-1}$, we get

$$
\begin{aligned}
\left|\varphi^{-1}(y)\right| & \leq \Theta_{\lambda_{k}}^{\lambda_{k+1}}(X) \leq 2^{m_{k}-m_{k-1}}=\operatorname{deg}_{m_{k-1}}^{m_{k}}\left(T_{2}\right) \\
& \leq \operatorname{deg}_{m_{k-1}}(y)=\left|\operatorname{pred}_{m_{k-1}}(y)\right|
\end{aligned}
$$

and consequently we can find a surjective map $\psi_{y}: \operatorname{pred}_{m_{k-1}}(y) \rightarrow \varphi_{n}^{-1}(y)$. By the choice of $\lambda_{k-1}$, for every $x \in \varphi_{k}^{-1}(y) \subset \lambda_{k}$ we get

$$
\begin{aligned}
\left|\operatorname{pred}_{\lambda_{k-1}}(x)\right|=\operatorname{deg}_{\lambda_{k-1}}(x) & \geq \operatorname{deg}_{\lambda_{k-1}}^{\lambda_{k}}\left(T_{X}\right) \\
& =\theta_{\lambda_{k-1}}^{\lambda_{k}}(X) \\
& \geq 2^{m_{k}-m_{k-1}} \\
& =\operatorname{Deg}_{m_{k-1}}^{m_{k}}\left(T_{2}\right) \\
& =\operatorname{deg}_{m_{k-1}}(y)=\left|\operatorname{pred}_{m_{k-1}}(y)\right| \geq\left|\psi_{y}^{-1}(x)\right|
\end{aligned}
$$

and so we can find a surjective map $\varphi_{x}: \operatorname{pred}_{\lambda_{k-1}}(x) \rightarrow \psi_{y}^{-1}(x)$. Now define the tower immersion $\varphi_{n-1}: T_{X}^{L_{k-1}} \rightarrow T_{2}^{M_{k-1}}$ by the formula

$$
\varphi_{k-1}=\varphi_{k} \cup \bigcup_{y \in m_{k}} \bigcup_{x \in \varphi_{k}^{-1}(y)} \varphi_{x} .
$$

After completing the inductive construction, we can see that

$$
\varphi=\bigcup_{n \leq 0} \varphi_{n}: T_{X}^{L} \rightarrow T_{2}^{M}
$$

is a tower immersion. By Proposition 5.4 (4), the tower immersion $\varphi$ induces a biuniform equivalence $\partial \varphi: \partial T_{X}^{L} \rightarrow \partial T_{2}^{M}$ between the boundaries of the towers $T_{X}^{L}$ and $T_{2}^{M}$, which are bi-uniformly equivalent to $X$ and $2^{<\mathbb{Z}}$, respectively.

\section{Proof of Theorem 4 (micro-uniform characterization of the Cantor bi-cube)}

The "only if" part of Theorem 4 easily follows from Lemmas 3.1 and 3.2. To prove the "if" part, it suffices to prove that any two non-compact complete metric spaces $X, Y$ of micro-uniform dimension zero are micro-uniformly equivalent if there is $\varepsilon \in(0,1)$ is such that $\Theta_{\delta}^{\varepsilon}(X)$ and $\Theta_{\delta}^{\varepsilon}(Y)$ are finite for all positive $\delta \leq \varepsilon$ and $\lim _{\delta \rightarrow+0} \theta_{\delta}^{\varepsilon}(X)=\infty=\lim _{\delta \rightarrow+0} \theta_{\delta}^{\varepsilon}(Y)$.

Being complete and not compact, the spaces $X$ and $Y$ are not totally bounded. Consequently, there is $\varepsilon_{0} \in(0,1)$ so small that $X$ cannot be covered by a finite number of sets of diameter $<\varepsilon_{0}$. Since $X$ has micro-uniform dimension zero, we can take a 
number $\varepsilon>0$ so small that each $\varepsilon$-connected component $C_{\varepsilon}(x), x \in X$, has diameter $<\varepsilon_{0}$. Then the choice of $\varepsilon_{0}$ guarantees that the cover $\mathcal{C}_{\varepsilon}(X)=\left\{C_{\varepsilon}(x) \mid x \in X\right\}$ is infinite. Since $X$ is separable the cover $\ell_{\varepsilon}(X)$ is countable.

For the same reason, we can assume that $\varepsilon$ is so small that $\mathcal{C}_{\varepsilon}(Y)=\left\{C_{\varepsilon}(y) \mid y \in Y\right\}$ is a countable cover of $Y$ consisting of sets of diameter $<\varepsilon_{0}$.

It is clear that the metric space $X$ is micro-uniformly equivalent to $X$ endowed with the metric $\min \left\{1, d_{X}\right\}$. So, we lose no generality assuming that $d_{X} \leq 1$. By the same reason, we can assume that $d_{Y} \leq 1$. In this case we prove that the bounded metric spaces $X, Y$ are bi-uniformly equivalent.

Let $\alpha_{0}=\beta_{0}=\varepsilon$ and $\alpha_{k}=\beta_{k}=k$ for $k \in \mathbb{N}$. By reverse induction, construct sequences $\left(\alpha_{k}\right)_{k=-\infty}^{-1}$ and $\left(\beta_{k}\right)_{k=-\infty}^{-1}$ of real numbers in the interval $(0,1)$ such that

(i) $\alpha_{k-1}<\alpha_{k}$ and $\beta_{k-1}<\beta_{k}$ for each $k \leq 0$,

(ii) $\lim _{k \rightarrow-\infty} \alpha_{k}=0, \lim _{k \rightarrow-\infty} \beta_{k}=0$,

(iii) $\theta_{\alpha_{k-1}}^{\alpha_{k}}(X) \geq \Theta_{\beta_{k-1}}^{\beta_{k}}(Y)$,

(iv) $\theta_{\beta_{k-1}}^{\beta_{k}}(Y) \geq \Theta_{\alpha_{k}}^{\alpha_{k+1}}(X)$.

For the level set $A=\left\{\alpha_{k} \mid k \in \mathbb{Z}\right\}$ consider the canonical $A$-tower $T_{X}^{A}=$ $\left\{\left(C_{\lambda}(x), \lambda\right) \mid x \in X, \lambda \in A\right\}$ of the metric space $X$. The level set $\operatorname{Lev}\left(T_{X}^{A}\right)$ of the tower $T_{X}^{A}$ can be identified with the set $A$. By Corollary 4.7 (3), the canonical map

$$
C_{A}: X \rightarrow \partial T_{X}^{A}, \quad C_{A}: x \mapsto C_{A}(x)=\left\{\left(C_{\lambda}(x), \lambda\right) \mid \lambda \in A\right\},
$$

is a bi-uniform equivalence. The choice of $\alpha_{0}=\varepsilon$ guarantees that the zeros level $\operatorname{Lev}_{0}\left(T_{X}^{A}\right)=\left\{\left(C_{\lambda}(x), \lambda\right) \mid x \in X, \lambda=\alpha_{0}\right\} \subset T_{X}^{A}$ is countable. On the other hand, $d_{X} \leq 1$ implies that for each $k \in \mathbb{N}$ the level $\operatorname{Lev}_{k}\left(T_{X}^{A}\right)=\left\{\left(C_{\alpha_{k}}(x), \alpha_{k}\right) \mid x \in X\right\}=$ $\{(X, k)\}$ is a singleton.

By analogy, for the level set $B=\left\{\beta_{k} \mid k \in \mathbb{Z}\right\}$ consider the canonical $B$-tower $T_{Y}^{B}=\left\{\left(C_{\lambda}(y), \lambda\right) \mid y \in Y, \lambda \in B\right\}$ of the metric space $Y$. By Corollary 4.7 (3), the canonical map

$$
C_{B}: Y \rightarrow \partial T_{Y}^{B}, \quad C_{B}: y \mapsto C_{B}(y)=\left\{\left(C_{\lambda}(y), \lambda\right) \mid \lambda \in B\right\},
$$

is a bi-uniform equivalence. The choice of $\beta_{0}=\varepsilon$ guarantees that the zeros level $\operatorname{Lev}_{0}\left(T_{Y}^{B}\right)=\left\{\left(C_{\lambda}(y), \lambda\right) \mid y \in Y, \lambda=\beta_{0}\right\} \subset T_{Y}^{B}$ is countable. On the other hand, $d_{Y} \leq 1$ implies that for each $k \in \mathbb{N}$ the level $\operatorname{Lev}_{k}\left(T_{Y}^{B}\right)=\left\{\left(C_{\beta_{k}}(y), \beta_{k}\right) \mid y \in\right.$ $Y\}=\{(Y, k)\}$ is a singleton.

For every $k \in \mathbb{Z}$ consider the sets $A_{k}=\left\{\alpha_{n} \mid n \geq k\right\}$ and $B_{k}=\left\{\alpha_{n} \mid n \geq k\right\}$. Let $\varphi_{1}: T_{X}^{A_{1}} \rightarrow T_{Y}^{B_{1}}$ be the tower isomorphism assigning to the unique point $(X, k)$ of a level of $T_{X}^{A_{0}}$ the unique point $(Y, k)$ of the corresponding level of the tower $T_{Y}^{B_{1}}$. Since the 0th levels of the towers $T_{X}^{A_{0}}$ and $T_{Y}^{B_{0}}$ both are countably infinite, we can extend the tower isomorphism $\varphi_{1}$ to a tower isomorphism $\varphi_{0}: T_{X}^{A_{0}} \rightarrow T_{Y}^{B_{0}}$.

By analogy with the proof of Theorem 5, by the reverse induction we can construct a sequence of surjective tower immersions $\varphi_{k}: T_{X}^{A_{k}} \rightarrow T_{Y}^{B_{k}}, k \leq 0$ such 
that $\varphi_{k-1} \mid T_{X}^{A_{k}}=\varphi_{k}$ for all $k \leq 0$. These tower immersions compose a surjective tower immersion $\varphi: T_{X}^{A} \rightarrow T_{Y}^{B}$ such that $\varphi \mid T_{X}^{A_{k}}=\varphi_{k}$ for all $k \leq 0$. By Proposition 5.4, the immersion $\varphi$ induces a micro-uniform equivalence $\partial \varphi: \partial T_{X}^{A} \rightarrow \partial T_{Y}^{B}$. By Corollary 4.7 (3), the boundary $\partial T_{X}^{A}$ is bi-uniformly equivalent to $X$ while $\partial T_{X}^{B}$ is bi-uniformly equivalent to $Y$. Consequently, the (bounded) metric spaces $X$ and $Y$ are bi-uniformly equivalent.

\section{Proof of Theorem 1 (the universality of the Cantor bi-cube)}

The "only if" part easily follows from Lemmas 3.1 and 3.2.

To prove the "if" part, assume that $X$ has bi-uniform dimension zero and $\Theta_{\delta}^{\varepsilon}(X)$ is finite for all $0<\delta<\varepsilon<\infty$. Since the completion of $X$ has the same properties, we lose no generality assuming that the space $X$ is complete.

For the level set $L=\left\{2^{n} \mid n \in \mathbb{Z}\right\}$ consider the canonical $L$-tower $T_{X}^{L}$ of $X$. By Corollary 4.7 (3), the canonical map $C_{L}: X \rightarrow \partial T_{X}^{L}$ is a bi-uniform equivalence. It follows that $\operatorname{Deg}_{2^{n}}^{2^{n+1}}\left(T_{X}^{L}\right)=\Theta_{2^{n}}^{2^{n+1}}(X)<\infty$ for all $2^{n} \in L=\operatorname{Lev}\left(T_{X}^{L}\right)$.

Let $T_{\omega}$ be a homogeneous tower such that the $\operatorname{set} \operatorname{Lev}\left(T_{\omega}\right)$ is order isomorphic to $\mathbb{Z}$ and $\operatorname{deg}(x)=\omega$ for each $x \in T$. Let $f: \operatorname{Lev}\left(T_{X}^{L}\right) \rightarrow \operatorname{Lev}\left(T_{\omega}\right)$ be an order isomorphism. By induction construct a homogeneous subtower $T \subset T_{\omega}$ such that

$$
\operatorname{Deg}_{\lambda}^{\lambda+1}(T)=\max \left\{2, \operatorname{Deg}_{f^{-1}(\lambda)}^{f^{-1}(\lambda+1)}\left(T_{X}\right)\right\} .
$$

By Proposition 5.8, there exists a tower embedding $\varphi: T_{X}^{L} \rightarrow T$ such that $\varphi_{\text {Lev }}=f$. By Proposition 5.4(2) the tower embedding $\varphi$ induces a bi-uniform embedding $\partial \varphi: \partial T_{X}^{L} \rightarrow \partial T$. By Theorem 6 , the boundary $\partial T$ of the homogeneous $\uparrow$-unbounded tower $T$ is bi-uniformly equivalent to the Cantor bi-cube $2^{<\mathbb{Z}}$. Since $X$ is bi-uniformly equivalent to $\partial T_{X}^{L}$, we see that $X$ bi-uniformly embeds into $2^{<\mathbb{Z}}$.

\section{Proof of Theorem 9}

Let $X$ be an isometrically homogeneous countable proper metric space of asymptotic dimension zero. Baire's theorem guarantees that $X$ has an isolated point and then the isometric homogeneity of $X$ implies that $X$ is uniformly discrete in the sense that for some $\varepsilon>0$ all $\varepsilon$-balls in $X$ are singletons. Being proper and uniformly discrete, the space $X$ is boundedly-finite. Since $X$ has asymptotic dimension zero, each $\varepsilon$-connected component $C_{\varepsilon}(x) \subset X$ is bounded and hence finite.

So, we can consider the function $f_{X}: \Pi \rightarrow \omega \cup\{\infty\}$ assigning to each prime number $p \in \Pi$ the (finite or infinite) number

$$
f_{X}(p)=\sup \left\{k \in \omega \mid p^{k} \text { divides }\left|C_{\varepsilon}(x)\right| \text { for some } \varepsilon>0 \text { and } x \in X\right\} .
$$


Given a function $f: \Pi \rightarrow \omega \cup\{\infty\}$ consider the direct sum

$$
\mathbb{Z}_{f}=\oplus_{p \in \Pi} \mathbb{Z}_{p}^{f(p)}
$$

of cyclic groups $\mathbb{Z}_{p}=\mathbb{Z} / p \mathbb{Z}$.

In [Sm] J. Smith proved that each countable group admits a proper left-invariant metric and that for any two proper left-invariant metrics $\rho, d$ on $G$ the identity map id: $(G, \rho) \rightarrow(G, d)$ is a bi-uniform equivalence. In the sequel we endow each countable group $G$ (in particular, each group $\mathbb{Z}_{f}$ ) with a proper left-invariant metric.

Lemma 11.1. Each isometrically homogeneous proper countable metric space $X$ of asymptotic dimension zero is bi-uniformly equivalent to the group $\mathbb{Z}_{f_{X}}$.

Proof. Consider the canonical $\omega$-tower $T_{X}^{\omega}=\left\{\left(C_{n}(x), n\right) \mid x \in X, n \in \omega\right\}$ of the metric space $X$.

Taking into account that each 0 -connected component $C_{0}(x)$ coincides with the singleton $\{x\}$ and applying Corollary 4.7, we conclude that canonical map $C_{\omega}: X \rightarrow$ $\partial T_{X}^{\omega}$ is a bi-uniform equivalence. The isometric homogeneity of the metric space $X$ implies the homogeneity of the tower $T_{X}^{\omega}$. It follows that for every $n \in \omega$ we the degree

$$
\operatorname{deg}_{n}\left(T_{X}^{\omega}\right)=\operatorname{deg}_{n}\left(T_{X}^{\omega}\right)=\left|C_{n+1}(x) / \mathcal{C}_{n}(X)\right|
$$

equals the number of $n$-connected components of $X$ composing an $(n+1)$-connected component of $X$.

For every $n \in \omega$ let $f_{n}: \Pi \rightarrow \omega$ be the function assigning to each prime number $p$ the maximal number $k \geq 0$ such that $p^{k}$ divides $\operatorname{deg}_{n}\left(T_{X}\right)$. Then the group $\mathbb{Z}_{f_{n}}$ is finite and has order $\left|\mathbb{Z}_{f_{n}}\right|=\operatorname{deg}_{n}\left(T_{X}\right)$.

Consider the group $G=\bigoplus_{n \in \omega} \mathbb{Z}_{f_{n}}$ and observe that it is isomorphic (with help of a coordinate permutating isomorphism) to the group $\mathbb{Z}_{f_{X}}$. The group $G$ can be written as the union $G=\bigcup_{m \in \omega} G_{m}$ of an increasing sequence $\left(G_{m}\right)_{m \in \omega}$ of subgroups where $G_{0}=\{0\}$ and $G_{m}=\bigcup_{n=0}^{m-1} \mathbb{Z}_{f_{n}}$ for $m>0$.

Consider the $\downarrow$-bounded tower $T_{G}=\left\{x G_{m} \mid x \in G, m \in \omega\right\}$ endowed with the inclusion relation and observe that it is homogeneous and $\operatorname{deg}_{n}\left(T_{G}\right)=$ $\left|\mathbb{Z}_{f_{n}}\right|=\operatorname{deg}_{n}\left(T_{X}\right)$ for all $n \in \omega$. By Proposition 5.8, there is a tower isomorphism $\varphi: T_{X}^{\omega} \rightarrow T_{G}$ inducing a bi-uniform equivalence $\partial \varphi: \partial T_{X}^{\omega} \rightarrow \partial T_{G}$. Then the bi-uniform equivalence between $X$ and $\mathbb{Z}_{f}$ is obtained as the composition of the bi-uniform equivalences:

$$
X \sim \partial T_{X}^{\omega} \sim \partial T_{G} \sim G \sim \mathbb{Z}_{f} .
$$

The following lemma (that essentially is due to I. Protasov [Pr]) combined with Lemma 11.1 implies Theorem 9.

Lemma 11.2. If two countable proper isometrically homogeneous metric spaces $X$, $Y$ of asymptotic dimension zero are bi-uniformly equivalent, then $f_{X}=f_{Y}$. 
Proof. Since $X$ and $Y$ are boundedly-finite spaces of asymptotic dimension zero their $\varepsilon$-connected components are finite for all $\varepsilon<\infty$.

The inequality $f_{X} \leq f_{Y}$ follows as soon as we check that for each prime number $p$ and each $k \in \mathbb{N}$ if $p^{k}$ divides the cardinality $\left|C_{\varepsilon}(x)\right|$ for some $x \in X$ and $\varepsilon<\infty$, then $p^{k}$ divides $\left|C_{\delta}(y)\right|$ for some $\delta<\infty$ and $y \in Y$.

Let $\varphi: X \rightarrow Y$ is a bi-uniform equivalence and $\delta=\omega_{\varphi}(\varepsilon)$. By Lemma 3.1, the image $\varphi\left(C_{\varepsilon}(x)\right)$ of the $\varepsilon$-connected component $C_{\varepsilon}(x)$ lies in the $\delta$-connected component $C_{\delta}(y)$ of the point $y=\varphi(x)$ in $Y$. Consider the preimage $A=\varphi^{-1}\left(C_{\delta}(y)\right)$ and observe that by Lemma 3.1 for each point $a \in A$ we get $\varphi\left(C_{\varepsilon}(a)\right) \subset C_{\delta}(\varphi(a))=$ $C_{\delta}(y)$ (the latter equality holds because $\varphi(a) \in C_{\delta}(y)$ ). Consequently, $C_{\varepsilon}(y) \subset A$. This implies that $A$ decomposes into a disjoint union of $\varepsilon$-connected components of $X$. Since the metric space $X$ is isometrically homogeneous, any two $\varepsilon$-connected component of $X$ have the same cardinality. Consequently, $\left|C_{\varepsilon}(x)\right|$ divides $|A|=\left|C_{\delta}(y)\right|$. Since $p^{k}$ divides $\left|C_{\varepsilon}(x)\right|$ it also divides $\left|C_{\delta}(y)\right|$. This concludes the proof of the inequality $f_{X} \leq f_{Y}$.

The inequality $f_{Y} \leq f_{X}$ can be proved by analogy.

Acknowledgment. The authors would like to express their sincere thanks to Igor Volodymyrovych Protasov and Jose Manuel Higes López for fruitful discussions and useful suggestions.

\section{References}

[BHZ] T. Banakh, J. Higes, and I. Zarichnyi, The coarse classification of countable abelian groups. Trans. Amer. Math. Soc. 362 (2010), 4755-4780. Zbl 05791769 MR 2645049

[BDHM] N. Brodskiy, J. Dydak, J. Higes, and A. Mitra, Dimension zero at all scales. Topology Appl. 154 (2007), 2729-2740. Zbl 1177.54015 MR 2340955

[DS] A. Dranishnikov and J. Smith, Asymptotic dimension of discrete groups. Fund. Math. 189 (2006), 27-34. Zbl 1100.20034 MR 2213160

[DZ] A. Dranishnikov and M. Zarichnyi, Universal spaces for asymptotic dimension. Topology Appl. 140 (2004), 203-225. Zbl 1063.54027 MR 2074917

[Hug] B. Hughes, Trees and ultrametric spaces: a categorical equivalence. Adv. Math. 189 (2004), 148-191. Zbl 1061.57021 MR 2093482

[Ke] A. S. Kechris, Classical descriptive set theory. Graduate Texts in Math. 156, Springer-Verlag, New York 1995. Zbl 0819.04002 MR 1321597

[MPM] Á. Martínez-Pérez and M. A. Morón, Uniformly continuous maps between ends of $\mathbb{R}$-trees. Math. Z. 263 (2009), 583-606. Zbl 1185.54029 MR 2545858

[Pr] I. V. Protasov, Morphisms of ball's structures of groups and graphs. Ukraïn. Mat. Zh. 54 (2002), 847-855; English transl. Ukrainian Math. J. 54 (2002), 1027-1037. Zbl 003.05053 MR 1956641 
[PB] I. Protasov and T. Banakh, Ball structures and colorings of graphs and groups. Math. Stud. Monogr. Ser. 11, VNTL Publ., Lviv 2003. Zbl 1147.05033 MR 2392704

[PZ] I. Protasov and M. Zarichnyi, General asymptology. Math. Stud. Monogr. Ser. 12, VNTL Publ., Lviv 2007. Zbl 1172.54002 MR 2406623

[Roe] J. Roe, Lectures on coarse geometry. Univ. Lecture Ser. 31, Amer. Math. Soc., Providence, RI, 2003. Zbl 1042.53027 MR 2007488

[Sj] J. Sanjurjo, Problems from the Madrid Department of Geometry and Topology. In Open problems in topology, II, Elsevier, Amsterdam 2007, 737-741.

[Sm] J. Smith, On asymptotic dimension of countable abelian groups. Topology Appl. 153 (2006), 2047-2054. Zbl 1144.20024 MR 2237596

Received October 5, 2009; revised June 8, 2010

T. Banakh, Instytut Matematyki, Uniwersytet Humanistyczno-Przyrodniczy Jana Kochanowskiego w Kielcach, Poland, and Faculty of Mechanics and Mathematics, Ivan Franko National University of Lviv, Universytetska 1, 79000, Lviv Ukraine

E-mail: tbanakh@yahoo.com; T.O.Banakh@gmail.com

I. Zarichnyi, Faculty of Mechanics and Mathematics, Ivan Franko National University of Lviv, ul. Universytets'ka 1, Lviv 79000, Ukraine, and Pidstryhach Institute for Applied Problems of Mechanics and Mathematics of National Academy of Sciences of Ukraine, Lviv, Naukova 3b, Ukraine

E-mail: ihor.zarichnyj@gmail.com 